Pure Appl. Chem., Vol. 81, No. 11, pp. 2131-2156, 2009.

doi:10.1351/PAC-REP-09-08-03

(C) 2009 IUPAC, Publication date (Web): 18 September 2009

\title{
Atomic weights of the elements 2007 (IUPAC Technical Report)*
}

\author{
Michael E. Wieser ${ }^{1, \ddagger}$ and Michael Berglund ${ }^{2}$ \\ ${ }^{1}$ Department of Physics and Astronomy, University of Calgary, Canada; ${ }^{2}$ European \\ Commission, Joint Research Centre, Institute for Reference Materials and \\ Measurements (IRMM), Belgium
}

\begin{abstract}
The latest evaluation of atomic weight determinations and other cognate data has warranted five changes for the standard atomic weights of the elements, $A_{\mathrm{r}}(\mathrm{E})$, from those published previously in the Table of Atomic Weights 2005. The revised standard atomic weight of nickel, $A_{\mathrm{r}}(\mathrm{Ni})$, is 58.6934(4); zinc, $A_{\mathrm{r}}(\mathrm{Zn})$, is 65.38(2); molybdenum, $A_{\mathrm{r}}(\mathrm{Mo})$, is 95.96(2); ytterbium, $A_{\mathrm{r}}(\mathrm{Yb})$, is 173.054(5); and lutetium, $A_{\mathrm{r}}(\mathrm{Lu})$, is $174.9668(1)$. Standard atomic weight tables abridged to four and five significant figures were also evaluated. The Commission-recommended value for the isotope-amount ratio of $n\left({ }^{40} \mathrm{Ar}\right) / n\left({ }^{36} \mathrm{Ar}\right)$, which is of importance in geochronology and geochemistry, has been changed to 298.56(31) from 296.03(53) based on new measurements. Atmospheric $\mathrm{O}_{2}$ is recognized as an international measurement standard, along with Vienna Standard Mean Ocean Water (VSMOW) and Vienna Pee Dee Belemnite (VPDB) carbonate for measurement and reporting of differences in relative oxygen isotope-amount ratios $\delta\left({ }^{17} \mathrm{O}\right)$ and $\delta\left({ }^{18} \mathrm{O}\right)$.
\end{abstract}

Keywords: argon isotopes; half-lives; IUPAC Inorganic Chemistry Division; lutetium; molybdenum; nickel; oxygen-17; ytterbium; zinc.

\section{INTRODUCTION}

The Commission on Isotopic Abundances and Atomic Weights (CIAAW) met in Pisa, Italy under the chairmanship of Prof. T. P. Ding from 30 to 31 July 2007, prior to the $44^{\text {th }}$ IUPAC General Assembly in Torino, Italy. The Commission decided to publish the report "Atomic Weights of the Elements 2007" as presented here. The resulting Table of Standard Atomic Weights is given in alphabetical order of the principal English names in Table 1 and in order of atomic number in Table 2. The standard atomic weights reported in Tables 1 and 2 are for atoms in their nuclear and electronic ground states.

At the 2007 meeting, the Commission reviewed recommendations of its Subcommittee on Isotopic Abundance Measurements (SIAM), which reviewed the literature from the past two years since the most recent published version of atomic weights [1,2]. The last complete compilation of isotopic compositions on an element-by-element basis was done in 2001 [3]. The SIAM data evaluation meeting was led under the chairmanship of Dr. M. Berglund.

The Commission periodically reviews the history of the standard atomic weight of each element, emphasizing the relevant published scientific evidence on which decisions have been made [4,5]. The Commission wishes to emphasize the need for new precise calibrated isotope composition measure-

\footnotetext{
*Sponsoring body: IUPAC Inorganic Chemistry Division, Commission on Isotopic Abundances and Atomic Weights: see more details on p. 2154.

¥Corresponding author: mwieser@ucalgary.ca
} 
ments in order to improve the standard atomic weights of a number of elements, which are still not known to a satisfactory level of accuracy. However, for many elements, the uncertainties of the standard atomic weights are limited by the variability among terrestrial materials rather than by the accuracy or precision of the measurements.

The atomic weight, $A_{\mathrm{r}}(\mathrm{E})$, of element $\mathrm{E}$ in a given substance can be determined from the knowledge of the isotopic abundances and corresponding atomic masses of the nuclides of that element. The Commission used the atomic mass evaluations of 2003 [6] for calculations of the atomic weights that were changed in this compilation. Depending on the element in question, there are several different types of decisions that may be needed to assign a standard atomic weight and uncertainty [7]. For mononuclidic elements like fluorine and phosphorus, the situation is relatively simple; the standard atomic weights are equal to the atomic masses as reported by the International Union of Pure and Applied Physics (IUPAP). In these cases, periodic changes to the values and uncertainties in atomic weights result from improved measurements of the atomic masses. For polyisotopic elements, the atomic weights may be different in different substances and the selection of the standard atomic weight is more complex. With minor exceptions to be covered by footnotes, the standard atomic weights and their uncertainties are intended to apply to almost all samples from natural terrestrial occurrences as well as to samples found in laboratories involved in chemical investigations, technological applications, or in materials of commerce.

In the recommendation of standard atomic weights, CIAAW generally has not attempted to estimate the average or composite isotopic composition of the Earth or of any subset of terrestrial materials. Instead, the Commission has attempted to find a single value and symmetrical uncertainty that would include almost all substances likely to be encountered, especially in the laboratory and in industry. Excluded from consideration in the standard atomic weights are most materials with deliberately altered isotopic compositions, extraterrestrial materials, and anomalous occurrences such as the Oklo natural nuclear reactor. The uncertainty $U\left[A_{\mathrm{r}}(\mathrm{E})\right]$ for a given standard atomic weight is given in parentheses following the last significant figure to which it is attributed in Tables 1,2, 4, and 5. If there is no specific statement on the distribution of possible values, then the distribution should be regarded as a rectangular distribution. The interval $A_{r}(\mathrm{E})-U\left[A_{\mathrm{r}}(\mathrm{E})\right]$ to $A_{\mathrm{r}}(\mathrm{E})+U\left[A_{\mathrm{r}}(\mathrm{E})\right]$ may be expected to encompass almost all samples from natural terrestrial occurrences as well as specimens and reagents found in laboratories involved in chemical investigations, technological applications, and in materials of commerce.

Variations in the relative amounts of isotopes of the elements in different materials commonly can be measured with greater precision than the ratios of the amounts of the isotopes (commonly termed an "absolute measurement"). For this reason, there are essentially four different categories of elements with contrasting constraints on their standard atomic weights:

1. mononuclidic

2. polynuclidic with no evidence for natural variation

3. polynuclidic with evidence of variation in the amounts of the isotopes within the uncertainties of the best absolute measurement

4. polynuclidic with variation in the amounts of the isotopes exceeding the uncertainties of the best absolute measurement

The footnote "r", referring to standard atomic weights whose uncertainties reflect variation, applies only to category 4 . Elements in category 3 may enter category 4 as more precise absolute determinations are made. Similarly, elements in category 2 can advance to category 3 as measurements improve. Within category 4, the footnote "g" refers to the subset of chemical elements for which the standard atomic weight and its uncertainties do not include all known variations. Thus, the footnotes "g" and "r" could occur together, or either one could occur alone.

For all elements for which a change in the value of $A_{\mathrm{r}}(\mathrm{E})$ or its uncertainty, $U\left[A_{\mathrm{r}}(\mathrm{E})\right]$ (in parentheses, following the last significant figure to which it is attributed) is recommended, the Commission 
by custom makes a statement on the reason for the change and includes a list of past recommended values over a period in excess of the last 100 years, which are taken from [7]. Values before the formation of the International Committee on Atomic Weights in 1900 come from [8].

The names and symbols for those elements with atomic numbers 112 to 118 referred to in the following tables, are systematic and based on the atomic numbers of the elements recommended for provisional use by the IUPAC publication "Nomenclature of Inorganic Chemistry" [9]. These systematic names and symbols will be replaced by a permanent name approved by IUPAC, once the priority of discovery is established and the name suggested by the discoverers is examined, reviewed, and accepted. The systematic name is derived directly from the atomic number of the element using the following numerical roots:

$$
\begin{array}{lllll}
1 \text { un } & 2 \text { bi } & 3 \text { tri } & 4 \text { quad } & 5 \text { pent } \\
6 \text { hex } & 7 \text { sept } & 8 \text { oct } & 9 \text { enn } & 0 \text { nil }
\end{array}
$$

The roots are put together in the order of the digits that make up the atomic number and terminated by "ium" to spell out the name. The final " $n$ " of "enn" is deleted when it occurs before "nil", and the "i" of "bi" and of "tri" is deleted when it occurs before "ium".

Table 1 Standard atomic weights 2007.

[Scaled to $\left.A_{\mathrm{r}}{ }^{12} \mathrm{C}\right)=12$, where ${ }^{12} \mathrm{C}$ is a neutral atom in its nuclear and electronic ground state.]

The atomic weights of many elements are not invariant, but depend on the origin and treatment of the material. The standard values of $A_{\mathrm{r}}(\mathrm{E})$ and the uncertainties (in parentheses, following the last significant figure to which they are attributed) apply to elements of natural terrestrial origin. The footnotes to this table elaborate the types of variation that may occur for individual elements and that may be larger than the listed uncertainties of values of $A_{r}(\mathrm{E})$. Names of elements with atomic numbers 112 to 118 are provisional.

\begin{tabular}{llcccc}
\hline \multicolumn{5}{c}{ Alphabetical order in English } & \\
\hline Element name & Symbol & Atomic number & Atomic weight & Footnotes \\
\hline actinium* & $\mathrm{Ac}$ & 89 & & & \\
aluminium (aluminum) & $\mathrm{Al}$ & 13 & $26.9815386(8)$ & & \\
americium* & $\mathrm{Am}$ & 95 & & & \\
antimony & $\mathrm{Sb}$ & 51 & $121.760(1)$ & $\mathrm{g}$ & \\
argon & $\mathrm{Ar}$ & 18 & $39.948(1)$ & $\mathrm{g}$ & $\mathrm{r}$ \\
arsenic & $\mathrm{As}$ & 33 & $74.92160(2)$ & & \\
astatine* & $\mathrm{At}$ & 85 & & & \\
barium & $\mathrm{Ba}$ & 56 & $137.327(7)$ & & \\
berkelium* & $\mathrm{Bk}$ & 97 & & & \\
beryllium & $\mathrm{Be}$ & 4 & $9.012182(3)$ & & \\
bismuth & $\mathrm{Bi}$ & 83 & $208.98040(1)$ & & \\
bohrium* & $\mathrm{Bh}$ & 107 & & & \\
boron & $\mathrm{B}$ & 5 & $10.811(7)$ & $\mathrm{g} \mathrm{m} \mathrm{r}$ \\
bromine & $\mathrm{Br}$ & 35 & $79.904(1)$ & & \\
cadmium & $\mathrm{Cd}$ & 48 & $112.411(8)$ & $\mathrm{g}$ & \\
caesium (cesium & $\mathrm{Cs}$ & 55 & $132.9054519(2)$ & & \\
calcium & $\mathrm{Ca}$ & 20 & $40.078(4)$ & $\mathrm{g}$ & \\
californium* & $\mathrm{Cf}$ & 98 & & & \\
carbon & $\mathrm{C}$ & 6 & $12.0107(8)$ & $\mathrm{g}$ & $\mathrm{r}$ \\
cerium & $\mathrm{Ce}$ & 58 & $140.116(1)$ & $\mathrm{g}$ & \\
chlorine & $\mathrm{Cl}$ & 17 & $35.453(2)$ & $\mathrm{g} \mathrm{m} \mathrm{r}$ \\
& & & & &
\end{tabular}


Table 1 (Continued).

\begin{tabular}{|c|c|c|c|c|c|}
\hline \multicolumn{6}{|c|}{ Alphabetical order in English } \\
\hline Element name & Symbol & Atomic number & Atomic weight & Footn & notes \\
\hline chromium & $\mathrm{Cr}$ & 24 & $51.9961(6)$ & & \\
\hline cobalt & Co & 27 & $58.933195(5)$ & & \\
\hline copper & $\mathrm{Cu}$ & 29 & $63.546(3)$ & & $\mathrm{r}$ \\
\hline curium* & $\mathrm{Cm}$ & 96 & & & \\
\hline darmstadtium* & Ds & 110 & & & \\
\hline dubnium* & $\mathrm{Db}$ & 105 & & & \\
\hline dysprosium & Dy & 66 & $162.500(1)$ & g & \\
\hline einsteinium* & Es & 99 & & & \\
\hline erbium & $\mathrm{Er}$ & 68 & $167.259(3)$ & $\mathrm{g}$ & \\
\hline europium & $\mathrm{Eu}$ & 63 & 151.964(1) & $\mathrm{g}$ & \\
\hline fermium* & Fm & 100 & & & \\
\hline fluorine & $\mathrm{F}$ & 9 & $18.9984032(5)$ & & \\
\hline francium* & $\mathrm{Fr}$ & 87 & & & \\
\hline gadolinium & $\mathrm{Gd}$ & 64 & $157.25(3)$ & $\mathrm{g}$ & \\
\hline gallium & $\mathrm{Ga}$ & 31 & $69.723(1)$ & & \\
\hline germanium & $\mathrm{Ge}$ & 32 & $72.64(1)$ & & \\
\hline gold & $\mathrm{Au}$ & 79 & $196.966569(4)$ & & \\
\hline hafnium & Hf & 72 & $178.49(2)$ & & \\
\hline hassium* & Hs & 108 & & & \\
\hline helium & $\mathrm{He}$ & 2 & $4.002602(2)$ & g & $\mathrm{r}$ \\
\hline holmium & Ho & 67 & $164.93032(2)$ & & \\
\hline hydrogen & $\mathrm{H}$ & 1 & $1.00794(7)$ & g m & $\mathrm{n} \mathrm{r}$ \\
\hline indium & In & 49 & $114.818(3)$ & & \\
\hline iodine & I & 53 & $126.90447(3)$ & & \\
\hline iridium & $\mathrm{Ir}$ & 77 & $192.217(3)$ & & \\
\hline iron & $\mathrm{Fe}$ & 26 & $55.845(2)$ & & \\
\hline krypton & $\mathrm{Kr}$ & 36 & $83.798(2)$ & $\mathrm{g} \mathrm{m}$ & \\
\hline lanthanum & $\mathrm{La}$ & 57 & $138.90547(7)$ & $\mathrm{g}$ & \\
\hline lawrencium* & $\mathrm{Lr}$ & 103 & & & \\
\hline lead & $\mathrm{Pb}$ & 82 & $207.2(1)$ & $\mathrm{g}$ & \\
\hline lithium & $\mathrm{Li}$ & 3 & {$[6.941(2)]^{\dagger}$} & $\mathrm{g} \mathrm{m}$ & $\mathrm{n} \mathrm{r}$ \\
\hline lutetium & $\mathrm{Lu}$ & 71 & $174.9668(1)$ & $\mathrm{g}$ & \\
\hline magnesium & $\mathrm{Mg}$ & 12 & $24.3050(6)$ & & \\
\hline manganese & $\mathrm{Mn}$ & 25 & $54.938045(5)$ & & \\
\hline meitnerium* & Mt & 109 & & & \\
\hline mendelevium* & Md & 101 & & & \\
\hline mercury & $\mathrm{Hg}$ & 80 & $200.59(2)$ & & \\
\hline molybdenum & Mo & 42 & $95.96(2)$ & $\mathrm{g}$ & \\
\hline neodymium & $\mathrm{Nd}$ & 60 & $144.242(3)$ & $\mathrm{g}$ & \\
\hline neon & $\mathrm{Ne}$ & 10 & $20.1797(6)$ & $\mathrm{g} \mathrm{m}$ & \\
\hline neptunium* & $\mathrm{Np}$ & 93 & & & \\
\hline nickel & $\mathrm{Ni}$ & 28 & $58.6934(4)$ & $\mathrm{r}$ & \\
\hline niobium & $\mathrm{Nb}$ & 41 & $92.90638(2)$ & & \\
\hline nitrogen & $\mathrm{N}$ & 7 & $14.0067(2)$ & g & $\mathrm{r}$ \\
\hline nobelium* & No & 102 & & & \\
\hline osmium & Os & 76 & $190.23(3)$ & g & \\
\hline oxygen & $\mathrm{O}$ & 8 & 15.9994(3) & $\mathrm{g}$ & $\mathrm{r}$ \\
\hline
\end{tabular}


Table 1 (Continued).

\begin{tabular}{|c|c|c|c|c|c|}
\hline \multicolumn{6}{|c|}{ Alphabetical order in English } \\
\hline Element name & Symbol & Atomic number & Atomic weight & \multicolumn{2}{|c|}{ Footnotes } \\
\hline palladium & $\mathrm{Pd}$ & 46 & $106.42(1)$ & \multicolumn{2}{|l|}{$\mathrm{g}$} \\
\hline phosphorus & $\mathrm{P}$ & 15 & $30.973762(2)$ & & \\
\hline platinum & $\mathrm{Pt}$ & 78 & 195.084(9) & & \\
\hline plutonium* & $\mathrm{Pu}$ & 94 & & & \\
\hline polonium* & Po & 84 & & & \\
\hline potassium & $\mathrm{K}$ & 19 & $39.0983(1)$ & & \\
\hline praseodymium & $\operatorname{Pr}$ & 59 & $140.90765(2)$ & & \\
\hline promethium* & $\mathrm{Pm}$ & 61 & & & \\
\hline protactinium* & $\mathrm{Pa}$ & 91 & $231.03588(2)$ & & \\
\hline radium* & $\mathrm{Ra}$ & 88 & & & \\
\hline radon* & $\mathrm{Rn}$ & 86 & & & \\
\hline roentgenium* & $\mathrm{Rg}$ & 111 & & & \\
\hline rhenium & $\operatorname{Re}$ & 75 & $186.207(1)$ & & \\
\hline rhodium & $\mathrm{Rh}$ & 45 & $102.90550(2)$ & & \\
\hline rubidium & $\mathrm{Rb}$ & 37 & $85.4678(3)$ & $\mathrm{g}$ & \\
\hline ruthenium & $\mathrm{Ru}$ & 44 & $101.07(2)$ & $\mathrm{g}$ & \\
\hline rutherfordium* & $\mathrm{Rf}$ & 104 & & & \\
\hline samarium & $\mathrm{Sm}$ & 62 & $150.36(2)$ & $\mathrm{g}$ & \\
\hline scandium & $\mathrm{Sc}$ & 21 & $44.955912(6)$ & & \\
\hline seaborgium* & $\mathrm{Sg}$ & 106 & & & \\
\hline selenium & $\mathrm{Se}$ & 34 & $78.96(3)$ & & $\mathrm{r}$ \\
\hline silicon & $\mathrm{Si}$ & 14 & $28.0855(3)$ & & $\mathrm{r}$ \\
\hline silver & $\mathrm{Ag}$ & 47 & $107.8682(2)$ & $\mathrm{g}$ & \\
\hline sodium & $\mathrm{Na}$ & 11 & $22.98976928(2)$ & & \\
\hline strontium & $\mathrm{Sr}$ & 38 & $87.62(1)$ & $\mathrm{g}$ & $\mathrm{r}$ \\
\hline sulfur & $\mathrm{S}$ & 16 & $32.065(5)$ & $\mathrm{g}$ & $\mathrm{r}$ \\
\hline tantalum & $\mathrm{Ta}$ & 73 & $180.94788(2)$ & & \\
\hline technetium* & $\mathrm{Tc}$ & 43 & & & \\
\hline tellurium & $\mathrm{Te}$ & 52 & $127.60(3)$ & $\mathrm{g}$ & \\
\hline terbium & $\mathrm{Tb}$ & 65 & $158.92535(2)$ & & \\
\hline thallium & $\mathrm{Tl}$ & 81 & $204.3833(2)$ & & \\
\hline thorium* & Th & 90 & $232.03806(2)$ & $\mathrm{g}$ & \\
\hline thulium & $\mathrm{Tm}$ & 69 & $168.93421(2)$ & & \\
\hline $\operatorname{tin}$ & $\mathrm{Sn}$ & 50 & $118.710(7)$ & $\mathrm{g}$ & \\
\hline titanium & $\mathrm{Ti}$ & 22 & $47.867(1)$ & & \\
\hline tungsten & W & 74 & $183.84(1)$ & & \\
\hline ununbium* & Uub & 112 & & & \\
\hline ununhexium* & Uuh & 116 & & & \\
\hline ununoctium* & Uuo & 118 & & & \\
\hline ununpentium* & Uup & 115 & & & \\
\hline ununquadium* & Uuq & 114 & & & \\
\hline ununtrium* & Uut & 113 & & & \\
\hline uranium* & $\mathrm{U}$ & 92 & $238.02891(3)$ & $\mathrm{g} 1$ & $\mathrm{~m}$ \\
\hline vanadium & V & 23 & $50.9415(1)$ & & \\
\hline xenon & $\mathrm{Xe}$ & 54 & $131.293(6)$ & $g_{1}$ & $\mathrm{~m}$ \\
\hline ytterbium & $\mathrm{Yb}$ & 70 & $173.054(5)$ & $\mathrm{g}$ & \\
\hline yttrium & $\mathrm{Y}$ & 39 & $88.90585(2)$ & & \\
\hline
\end{tabular}


Table 1 (Continued).

\begin{tabular}{lcccc}
\hline \multicolumn{5}{c}{ Alphabetical order in English } \\
\hline Element name & Symbol & Atomic number & Atomic weight & Footnotes \\
\hline zinc & $\mathrm{Zn}$ & 30 & $65.38(2)$ & $\mathrm{r}$ \\
zirconium & $\mathrm{Zr}$ & 40 & $91.224(2)$ & $\mathrm{g}$ \\
\hline
\end{tabular}

*Element has no stable nuclides. One or more well-known isotopes are given in Table 3 with the appropriate relative atomic mass and half-life. However, three such elements (Th, $\mathrm{Pa}$, and $\mathrm{U}$ ) do have a characteristic terrestrial isotopic composition, and for these an atomic weight is tabulated.

${ }^{\dagger}$ Commercially available Li materials have atomic weights that range between 6.939 and 6.996; if a more accurate value is required, it must be determined for the specific material.

g Geological specimens are known in which the element has an isotopic composition outside the limits for normal material. The difference between the atomic weight of the element in such specimens and that given in the table may exceed the stated uncertainty.

$\mathrm{m} \quad$ Modified isotopic compositions may be found in commercially available material because it has been subjected to an undisclosed or inadvertent isotopic fractionation. Substantial deviations in atomic weight of the element from that given in the table can occur.

$\mathrm{r} \quad$ Range in isotopic composition of normal terrestrial material prevents a more precise $A_{\mathrm{r}}(\mathrm{E})$ being given; the tabulated $A_{\mathrm{r}}(\mathrm{E})$ value and uncertainty should be applicable to normal material.

Table 2 Standard atomic weights 2007.

[Scaled to $\left.A_{r}{ }^{12} \mathrm{C}\right)=12$, where ${ }^{12} \mathrm{C}$ is a neutral atom in its nuclear and electronic ground state.]

The atomic weights of many elements are not invariant, but depend on the origin and treatment of the material. The standard values of $A_{r}(\mathrm{E})$ and the uncertainties (in parentheses, following the last significant figure to which they are attributed) apply to elements of natural terrestrial origin. The footnotes to this table elaborate the types of variation that may occur for individual elements and that may be larger than the listed uncertainties of values of $A_{r}(\mathrm{E})$. Names of elements with atomic number 112 to 118 are provisional.

\begin{tabular}{|c|c|c|c|c|c|}
\hline \multicolumn{6}{|c|}{ Order of atomic number } \\
\hline Atomic number & Element name & Symbol & Atomic weight & Footnc & hotes \\
\hline 1 & hydrogen & $\mathrm{H}$ & $1.00794(7)$ & g m & $\mathrm{n} \mathrm{r}$ \\
\hline 2 & helium & $\mathrm{He}$ & $4.002602(2)$ & $\mathrm{g}$ & $\mathrm{r}$ \\
\hline 3 & lithium & $\mathrm{Li}$ & {$[6.941(2)]^{\dagger}$} & $\mathrm{g} \mathrm{m}$ & $\mathrm{n} \mathrm{r}$ \\
\hline 4 & beryllium & $\mathrm{Be}$ & $9.012182(3)$ & & \\
\hline 5 & boron & B & $10.811(7)$ & g m & $\mathrm{n} \mathrm{r}$ \\
\hline 6 & carbon & $\mathrm{C}$ & $12.0107(8)$ & $\mathrm{g}$ & $\mathrm{r}$ \\
\hline 7 & nitrogen & $\mathrm{N}$ & $14.0067(2)$ & g & $\mathrm{r}$ \\
\hline 8 & oxygen & $\mathrm{O}$ & $15.9994(3)$ & $\mathrm{g}$ & $\mathrm{r}$ \\
\hline 9 & fluorine & $\mathrm{F}$ & $18.9984032(5)$ & & \\
\hline 10 & neon & $\mathrm{Ne}$ & $20.1797(6)$ & $\mathrm{g} \mathrm{m}$ & \\
\hline 11 & sodium & $\mathrm{Na}$ & $22.98976928(2)$ & & \\
\hline 12 & magnesium & $\mathrm{Mg}$ & $24.3050(6)$ & & \\
\hline 13 & aluminium (aluminum) & $\mathrm{Al}$ & $26.9815386(8)$ & & \\
\hline 14 & silicon & $\mathrm{Si}$ & $28.0855(3)$ & & $\mathrm{r}$ \\
\hline 15 & phosphorus & $\mathrm{P}$ & $30.973762(2)$ & & \\
\hline 16 & sulfur & S & $32.065(5)$ & & $\mathrm{r}$ \\
\hline 17 & chlorine & $\mathrm{Cl}$ & $35.453(2)$ & g m & $\mathrm{n} \mathrm{r}$ \\
\hline 18 & argon & $\mathrm{Ar}$ & $39.948(1)$ & $\mathrm{g}$ & $\mathrm{r}$ \\
\hline 19 & potassium & $\mathrm{K}$ & 39.0983(1) & & \\
\hline
\end{tabular}

(continues on next page) 
Table 2 (Continued).

\begin{tabular}{|c|c|c|c|c|c|}
\hline \multicolumn{6}{|c|}{ Order of atomic number } \\
\hline Atomic number & Element name & Symbol & Atomic weight & Footr & notes \\
\hline 20 & calcium & $\mathrm{Ca}$ & $40.078(4)$ & $\mathrm{g}$ & \\
\hline 21 & scandium & $\mathrm{Sc}$ & $44.955912(6)$ & & \\
\hline 22 & titanium & $\mathrm{Ti}$ & $47.867(1)$ & & \\
\hline 23 & vanadium & $\mathrm{V}$ & $50.9415(1)$ & & \\
\hline 24 & chromium & $\mathrm{Cr}$ & $51.9961(6)$ & & \\
\hline 25 & manganese & Mn & $54.938045(5)$ & & \\
\hline 26 & iron & $\mathrm{Fe}$ & $55.845(2)$ & & \\
\hline 27 & cobalt & Co & $58.933195(5)$ & & \\
\hline 28 & nickel & $\mathrm{Ni}$ & $58.6934(4)$ & & $\mathrm{r}$ \\
\hline 29 & copper & $\mathrm{Cu}$ & $63.546(3)$ & & $\mathrm{r}$ \\
\hline 30 & zinc & $\mathrm{Zn}$ & $65.38(2)$ & & $\mathrm{r}$ \\
\hline 31 & gallium & $\mathrm{Ga}$ & $69.723(1)$ & & \\
\hline 32 & germanium & $\mathrm{Ge}$ & $72.64(1)$ & & \\
\hline 33 & arsenic & As & $74.92160(2)$ & & \\
\hline 34 & selenium & $\mathrm{Se}$ & $78.96(3)$ & & $\mathrm{r}$ \\
\hline 35 & bromine & $\mathrm{Br}$ & 79.904(1) & & \\
\hline 36 & krypton & $\mathrm{Kr}$ & $83.798(2)$ & $\mathrm{g} \mathrm{n}$ & \\
\hline 37 & rubidium & $\mathrm{Rb}$ & $85.4678(3)$ & $\mathrm{g}$ & \\
\hline 38 & strontium & $\mathrm{Sr}$ & $87.62(1)$ & $\mathrm{g}$ & $\mathrm{r}$ \\
\hline 39 & yttrium & $\mathrm{Y}$ & $88.90585(2)$ & & \\
\hline 40 & zirconium & $\mathrm{Zr}$ & $91.224(2)$ & $\mathrm{g}$ & \\
\hline 41 & niobium & $\mathrm{Nb}$ & $92.90638(2)$ & & \\
\hline 42 & molybdenum & Mo & $95.96(2)$ & $\mathrm{g}$ & $\mathrm{r}$ \\
\hline 43 & technetium* & $\mathrm{Tc}$ & & & \\
\hline 44 & ruthenium & $\mathrm{Ru}$ & $101.07(2)$ & $\mathrm{g}$ & \\
\hline 45 & rhodium & $\mathrm{Rh}$ & $102.90550(2)$ & & \\
\hline 46 & palladium & $\mathrm{Pd}$ & $106.42(1)$ & $\mathrm{g}$ & \\
\hline 47 & silver & $\mathrm{Ag}$ & $107.8682(2)$ & $\mathrm{g}$ & \\
\hline 48 & cadmium & $\mathrm{Cd}$ & $112.411(8)$ & $\mathrm{g}$ & \\
\hline 49 & indium & In & $114.818(3)$ & & \\
\hline 50 & tin & $\mathrm{Sn}$ & $118.710(7)$ & $\mathrm{g}$ & \\
\hline 51 & antimony & $\mathrm{Sb}$ & $121.760(1)$ & $\mathrm{g}$ & \\
\hline 52 & tellurium & $\mathrm{Te}$ & $127.60(3)$ & $\mathrm{g}$ & \\
\hline 53 & iodine & I & $126.90447(3)$ & & \\
\hline 54 & xenon & $\mathrm{Xe}$ & $131.293(6)$ & $\mathrm{g} \mathrm{n}$ & \\
\hline 55 & caesium (cesium) & Cs & $132.9054519(2)$ & & \\
\hline 56 & barium & $\mathrm{Ba}$ & 137.327(7) & & \\
\hline 57 & lanthanum & $\mathrm{La}$ & $138.90547(7)$ & $\mathrm{g}$ & \\
\hline 58 & cerium & $\mathrm{Ce}$ & $140.116(1)$ & $\mathrm{g}$ & \\
\hline 59 & praseodymium & $\operatorname{Pr}$ & $140.90765(2)$ & & \\
\hline 60 & neodymium & $\mathrm{Nd}$ & $144.242(3)$ & $\mathrm{g}$ & \\
\hline 61 & promethium* & $\mathrm{Pm}$ & & & \\
\hline 62 & samarium & $\mathrm{Sm}$ & $150.36(2)$ & $\mathrm{g}$ & \\
\hline 63 & europium & $\mathrm{Eu}$ & $151.964(1)$ & $\mathrm{g}$ & \\
\hline 64 & gadolinium & Gd & $157.25(3)$ & $\mathrm{g}$ & \\
\hline 65 & terbium & $\mathrm{Tb}$ & $158.92535(2)$ & & \\
\hline 66 & dysprosium & Dy & $162.500(1)$ & $\mathrm{g}$ & \\
\hline 67 & holmium & Ho & $164.93032(2)$ & & \\
\hline
\end{tabular}

(continues on next page) 
Table 2 (Continued).

\begin{tabular}{|c|c|c|c|c|}
\hline \multicolumn{5}{|c|}{ Order of atomic number } \\
\hline Atomic number & Element name & Symbol & Atomic weight & Footnotes \\
\hline 68 & erbium & $\mathrm{Er}$ & $167.259(3)$ & $\mathrm{g}$ \\
\hline 69 & thulium & $\mathrm{Tm}$ & $168.93421(2)$ & \\
\hline 70 & ytterbium & $\mathrm{Yb}$ & $173.054(5)$ & $\mathrm{g}$ \\
\hline 71 & lutetium & $\mathrm{Lu}$ & $174.9668(1)$ & $\mathrm{g}$ \\
\hline 72 & hafnium & Hf & $178.49(2)$ & \\
\hline 73 & tantalum & $\mathrm{Ta}$ & $180.94788(2)$ & \\
\hline 74 & tungsten & $\mathrm{W}$ & $183.84(1)$ & \\
\hline 75 & rhenium & $\mathrm{Re}$ & $186.207(1)$ & \\
\hline 76 & osmium & Os & $190.23(3)$ & $\mathrm{g}$ \\
\hline 77 & iridium & $\mathrm{Ir}$ & $192.217(3)$ & \\
\hline 78 & platinum & $\mathrm{Pt}$ & 195.084(9) & \\
\hline 79 & gold & $\mathrm{Au}$ & $196.966569(4)$ & \\
\hline 80 & mercury & $\mathrm{Hg}$ & $200.59(2)$ & \\
\hline 81 & thallium & $\mathrm{Tl}$ & $204.3833(2)$ & \\
\hline 82 & lead & $\mathrm{Pb}$ & 207.2(1) & $\begin{array}{ll}\mathrm{g} & \mathrm{r}\end{array}$ \\
\hline 83 & bismuth & $\mathrm{Bi}$ & $208.98040(1)$ & \\
\hline 84 & polonium* & Po & & \\
\hline 85 & astatine* & At & & \\
\hline 86 & radon* & $\mathrm{Rn}$ & & \\
\hline 87 & francium* & $\mathrm{F}$ & & \\
\hline 88 & radium* & $\mathrm{Ra}$ & & \\
\hline 89 & actinium* & $\mathrm{Ac}$ & & \\
\hline 90 & thorium* & Th & $232.03806(2)$ & $\mathrm{g}$ \\
\hline 91 & protactinium* & $\mathrm{Pa}$ & $231.03588(2)$ & \\
\hline 92 & uranium* & $\mathrm{U}$ & $238.02891(3)$ & $\mathrm{g} \mathrm{m}$ \\
\hline 93 & neptunium* & $\mathrm{Np}$ & & \\
\hline 94 & plutonium* & $\mathrm{Pu}$ & & \\
\hline 95 & americium* & Am & & \\
\hline 96 & curium* & $\mathrm{Cm}$ & & \\
\hline 97 & berkelium* & $\mathrm{Bk}$ & & \\
\hline 98 & californium* & $\mathrm{Cf}$ & & \\
\hline 99 & einsteinium* & Es & & \\
\hline 100 & fermium* & $\mathrm{Fm}$ & & \\
\hline 101 & mendelevium* & Md & & \\
\hline 102 & nobelium* & No & & \\
\hline 103 & lawrencium* & $\mathrm{Lr}$ & & \\
\hline 104 & rutherfordium* & $\mathrm{Rf}$ & & \\
\hline 105 & dubnium* & $\mathrm{Db}$ & & \\
\hline 106 & seaborgium* & $\mathrm{Sg}$ & & \\
\hline 107 & bohrium* & $\mathrm{Bh}$ & & \\
\hline 108 & hassium* & $\mathrm{Hs}$ & & \\
\hline 109 & meitnerium* & Mt & & \\
\hline 110 & darmstadtium* & Ds & & \\
\hline 111 & roentgenium* & $\mathrm{Rg}$ & & \\
\hline 112 & ununbium* & Uub & & \\
\hline 113 & ununtrium* & Uut & & \\
\hline 114 & ununquadium* & Uuq & & \\
\hline 115 & ununpentium* & Uup & & \\
\hline
\end{tabular}

(continues on next page) 
Table 2 (Continued).

\begin{tabular}{llccl}
\hline \multicolumn{4}{c}{ Order of atomic number } \\
\hline Atomic number & Element name & Symbol & Atomic weight & Footnotes \\
\hline 116 & ununhexium* & Uuh & & \\
118 & ununoctium* & Uuo & & \\
\hline
\end{tabular}

*Element has no stable nuclides. One or more well-known isotopes are given in Table 3 with the appropriate relative atomic mass and half-life. However, three such elements (Th, $\mathrm{Pa}$, and $\mathrm{U}$ ) do have a characteristic terrestrial isotopic composition, and for these an atomic weight is tabulated.

${ }^{\dagger}$ Commercially available Li materials have atomic weights that range between 6.939 and 6.996; if a more accurate value is required, it must be determined for the specific material.

g Geological specimens are known in which the element has an isotopic composition outside the limits for normal material. The difference between the atomic weight of the element in such specimens and that given in the table may exceed the stated uncertainty.

$\mathrm{m} \quad$ Modified isotopic compositions may be found in commercially available material because it has been subjected to an undisclosed or inadvertent isotopic fractionation. Substantial deviations in atomic weight of the element from that given in the table can occur.

$\mathrm{r} \quad$ Range in isotopic composition of normal terrestrial material prevents a more precise $A_{\mathrm{r}}(\mathrm{E})$ being given; the tabulated $A_{\mathrm{r}}(\mathrm{E})$ value and uncertainty should be applicable to normal material.

Table 3 Relative atomic masses and half-lives of selected radionuclides. Listing of particular nuclides for elements numbered 112 and above in Table 3 does not imply any priority of the discovery of those elements on the part of the Commission.

[Prepared, as in previous years, by N. E. Holden, a former Commission member; $\mathrm{a}=$ year; $\mathrm{d}=$ day; $\mathrm{h}=$ hour; $\min =$ minute; $\mathrm{s}=$ second. Names of elements with atomic number 112 to 118 are provisional.]

\begin{tabular}{|c|c|c|c|c|c|c|}
\hline $\begin{array}{l}\text { Atomic } \\
\text { number }\end{array}$ & $\begin{array}{c}\text { Element } \\
\text { name }\end{array}$ & Symbol & $\begin{array}{c}\text { Mass } \\
\text { number }\end{array}$ & Atomic mass & Half-life & Unit \\
\hline \multirow[t]{3}{*}{43} & technetium & $\mathrm{Tc}$ & 97 & 96.9064 & $4.21(16) \times 10^{6}$ & $a$ \\
\hline & & & 98 & 97.9072 & $6.6(10) \times 10^{6}$ & $\mathrm{a}$ \\
\hline & & & 99 & 98.9063 & $2.1(3) \times 10^{5}$ & $\mathrm{a}$ \\
\hline \multirow[t]{3}{*}{61} & promethium & $\mathrm{Pm}$ & 145 & 144.9127 & $17.7(4)$ & $\mathrm{a}$ \\
\hline & & & 146 & 145.9147 & $5.53(5)$ & $\mathrm{a}$ \\
\hline & & & 147 & 146.9151 & $2.623(3)$ & $\mathrm{a}$ \\
\hline \multirow[t]{3}{*}{84} & polonium & Po & 208 & 207.9812 & $2.90(1)$ & $\mathrm{a}$ \\
\hline & & & 209 & 208.9824 & $1.3(2) \times 10^{2}$ & $\mathrm{a}$ \\
\hline & & & 210 & 209.9829 & $138.4(1)$ & d \\
\hline \multirow[t]{2}{*}{85} & astatine & At & 210 & 209.9871 & $8.1(4)$ & $\mathrm{h}$ \\
\hline & & & 211 & 210.9875 & $7.21(1)$ & $\mathrm{h}$ \\
\hline \multirow[t]{3}{*}{86} & radon & $\mathrm{Rn}$ & 210 & 209.9897 & $2.4(1)$ & $\mathrm{h}$ \\
\hline & & & 211 & 210.9906 & $14.6(2)$ & $\mathrm{h}$ \\
\hline & & & 222 & 222.0176 & $3.823(4)$ & d \\
\hline \multirow[t]{3}{*}{87} & francium & $\mathrm{Fr}$ & 212 & 211.9962 & $20.0(6)$ & $\min$ \\
\hline & & & 222 & 222.0176 & $14.2(3)$ & $\min$ \\
\hline & & & 223 & 223.0197 & $22.0(1)$ & $\min$ \\
\hline \multirow[t]{2}{*}{88} & radium & $\mathrm{Ra}$ & 226 & 226.0254 & $1599(4)$ & $\mathrm{a}$ \\
\hline & & & 228 & 228.0311 & $5.76(3)$ & $\mathrm{a}$ \\
\hline \multirow[t]{2}{*}{89} & actinium & Ac & 225 & 225.0232 & $10.0(1)$ & $\mathrm{d}$ \\
\hline & & & 227 & 227.0278 & $21.77(2)$ & $\mathrm{a}$ \\
\hline \multirow[t]{2}{*}{90} & thorium & Th & 230 & 230.0331 & $7.54(3) \times 10^{6}$ & $\mathrm{a}$ \\
\hline & & & 232 & 232.0381 & $1.40(1) \times 10^{10}$ & $\mathrm{a}$ \\
\hline
\end{tabular}

(continues on next page) 
Table 3 (Continued).

\begin{tabular}{|c|c|c|c|c|c|c|}
\hline $\begin{array}{l}\text { Atomic } \\
\text { number }\end{array}$ & $\begin{array}{l}\text { Element } \\
\text { name }\end{array}$ & Symbol & $\begin{array}{c}\text { Mass } \\
\text { number }\end{array}$ & Atomic mass & Half-life & Unit \\
\hline \multirow[t]{2}{*}{91} & \multirow[t]{2}{*}{ protactinium } & \multirow[t]{2}{*}{$\mathrm{Pa}$} & 231 & 231.0359 & $3.25(1) \times 10^{4}$ & $\mathrm{a}$ \\
\hline & & & 233 & 233.04025 & $27.0(1)$ & $\mathrm{d}$ \\
\hline \multirow[t]{5}{*}{92} & \multirow[t]{5}{*}{ uranium } & \multirow[t]{5}{*}{$\mathrm{U}$} & 233 & 233.0396 & $1.592(2) \times 10^{5}$ & $\mathrm{a}$ \\
\hline & & & 234 & 234.0410 & $2.455(6) \times 10^{5}$ & $\mathrm{a}$ \\
\hline & & & 235 & 235.0439 & $7.04(1) \times 10^{8}$ & $\mathrm{a}$ \\
\hline & & & 236 & 236.0456 & $2.342(4) \times 10^{7}$ & $\mathrm{a}$ \\
\hline & & & 238 & 238.0508 & $4.468(3) \times 10^{9}$ & $\mathrm{a}$ \\
\hline \multirow[t]{2}{*}{93} & \multirow[t]{2}{*}{ neptunium } & \multirow[t]{2}{*}{$\mathrm{Np}$} & 236 & 236.0466 & $1.54(6) \times 10^{5}$ & $\mathrm{a}$ \\
\hline & & & 237 & 237.0482 & $2.14(1) \times 10^{6}$ & $\mathrm{a}$ \\
\hline \multirow[t]{6}{*}{94} & \multirow[t]{6}{*}{ plutonium } & \multirow[t]{6}{*}{$\mathrm{Pu}$} & 238 & 238.0496 & $87.7(1)$ & $\mathrm{a}$ \\
\hline & & & 239 & 239.0522 & $2.410(3) \times 10^{4}$ & $\mathrm{a}$ \\
\hline & & & 240 & 240.0538 & $6.56(1) \times 10^{3}$ & $\mathrm{a}$ \\
\hline & & & 241 & 241.0569 & $14.4(1)$ & $\mathrm{a}$ \\
\hline & & & 242 & 242.0587 & $3.75(2) \times 10^{5}$ & $\mathrm{a}$ \\
\hline & & & 244 & 244.0642 & $8.00(9) \times 10^{7}$ & $\mathrm{a}$ \\
\hline \multirow[t]{2}{*}{95} & \multirow[t]{2}{*}{ americium } & \multirow[t]{2}{*}{$\mathrm{Am}$} & 241 & 241.0568 & $432.7(6)$ & $\mathrm{a}$ \\
\hline & & & 243 & 243.0614 & $7.37(2) \times 10^{3}$ & $\mathrm{a}$ \\
\hline \multirow[t]{6}{*}{96} & \multirow[t]{6}{*}{ curium } & \multirow[t]{6}{*}{$\mathrm{Cm}$} & 243 & 243.0614 & 29.1(1) & $\mathrm{a}$ \\
\hline & & & 244 & 244.0628 & $18.1(1)$ & $\mathrm{a}$ \\
\hline & & & 245 & 245.0655 & $8.48(6) \times 10^{3}$ & $\mathrm{a}$ \\
\hline & & & 246 & 246.0672 & $4.76(4) \times 10^{3}$ & $\mathrm{a}$ \\
\hline & & & 247 & 247.0704 & $1.56(5) \times 10^{7}$ & $\mathrm{a}$ \\
\hline & & & 248 & 248.0723 & $3.48(6) \times 10^{5}$ & $\mathrm{a}$ \\
\hline \multirow[t]{2}{*}{97} & \multirow[t]{2}{*}{ berkelium } & \multirow[t]{2}{*}{$\mathrm{Bk}$} & 247 & 247.0703 & $1.4(3) \times 10^{3}$ & $\mathrm{a}$ \\
\hline & & & 249 & 249.0750 & $3.20(3) \times 10^{2}$ & $\mathrm{~d}$ \\
\hline 98 & californium & $\mathrm{Cf}$ & 249 & 249.0749 & $351(2)$ & $\mathrm{a}$ \\
\hline & & & 250 & 250.0764 & $13.1(1)$ & $\mathrm{a}$ \\
\hline & & & 251 & 251.0796 & $9.0(5) \times 10^{2}$ & $\mathrm{a}$ \\
\hline & & & 252 & 252.0816 & $2.65(1)$ & $\mathrm{a}$ \\
\hline 99 & einsteinium & Es & 252 & 252.0830 & $472(2)$ & $\mathrm{d}$ \\
\hline & & & 254 & 254.0880 & $276(1)$ & $\mathrm{d}$ \\
\hline 100 & fermium & $\mathrm{Fm}$ & 253 & 253.0852 & $3.0(1)$ & $\mathrm{d}$ \\
\hline & & & 257 & 257.0951 & $100.5(2)$ & $\mathrm{d}$ \\
\hline 101 & mendelevium & Md & 258 & 258.0984 & $51.5(3)$ & $\mathrm{d}$ \\
\hline & & & 260 & 260.1037 & $27.8(3)$ & $\mathrm{d}$ \\
\hline 102 & nobelium & No & 255 & 255.0932 & $3.1(2)$ & $\min$ \\
\hline & & & 259 & 259.1010 & $58(5)$ & $\min$ \\
\hline 103 & lawrencium & $\mathrm{Lr}$ & 251 & 251.0944 & $\sim 39$ & $\min$ \\
\hline & & & 261 & 261.1069 & $\sim 40$ & $\min$ \\
\hline & & & 262 & 262.1096 & $3.6(3)$ & $\mathrm{h}$ \\
\hline 104 & rutherfordium & $\mathrm{Rf}$ & 265 & 265.1167 & 13 & $\mathrm{~h}$ \\
\hline & & & 267 & 267.122 & $\sim 1$ & $\mathrm{~h}$ \\
\hline 105 & dubnium & $\mathrm{Db}$ & 267 & 267.1224 & $\sim 1.2$ & $\mathrm{~h}$ \\
\hline & & & 268 & 268.125 & $1.2(4)$ & $\mathrm{d}$ \\
\hline 106 & seaborgium & $\mathrm{Sg}$ & 265 & 265.1211 & $\sim 15$ & $\mathrm{~s}$ \\
\hline & & & 271 & 271.133 & $\sim 2$ & $\min$ \\
\hline 107 & bohrium & $\mathrm{Bh}$ & 267 & 267.1277 & $\sim 17$ & $\mathrm{~s}$ \\
\hline & & & 272 & & $\sim 1$ & $\min$ \\
\hline
\end{tabular}

(continues on next page) 
Table 3 (Continued).

\begin{tabular}{|c|c|c|c|c|c|c|}
\hline $\begin{array}{l}\text { Atomic } \\
\text { number }\end{array}$ & $\begin{array}{c}\text { Element } \\
\text { name }\end{array}$ & Symbol & $\begin{array}{c}\text { Mass } \\
\text { number }\end{array}$ & Atomic mass & Half-life & Unit \\
\hline \multirow[t]{2}{*}{108} & \multirow[t]{2}{*}{ hassium } & \multirow[t]{2}{*}{ Hs } & 269 & 269.1341 & $\sim 10$ & $\mathrm{~s}$ \\
\hline & & & 277 & 277.150 & 11 & $\min$ \\
\hline \multirow[t]{2}{*}{109} & \multirow[t]{2}{*}{ meitnerium } & \multirow[t]{2}{*}{ Mt } & 268 & 268.1387 & $\sim 0.03$ & $\mathrm{~s}$ \\
\hline & & & 276 & 276.151 & $\sim 0.7$ & $\mathrm{~s}$ \\
\hline \multirow[t]{2}{*}{110} & \multirow[t]{2}{*}{ darmstadtium } & \multirow[t]{2}{*}{ Ds } & 280 & 280.160 & $\sim 7.6$ & $\mathrm{~s}$ \\
\hline & & & 281 & 281.162 & $\sim 11$ & $\mathrm{~s}$ \\
\hline \multirow[t]{2}{*}{111} & \multirow[t]{2}{*}{ roentgenium } & \multirow[t]{2}{*}{$\mathrm{Rg}$} & 279 & 279.162 & $\sim 0.17$ & $\mathrm{~s}$ \\
\hline & & & 280 & 280.164 & $\sim 3.6$ & $\mathrm{~s}$ \\
\hline \multirow[t]{2}{*}{112} & \multirow[t]{2}{*}{ ununbium } & \multirow[t]{2}{*}{ Uub } & 283 & 283.172 & $\sim 4$ & $\mathrm{~s}$ \\
\hline & & & 285 & 285.174 & $\sim 29$ & $\mathrm{~s}$ \\
\hline \multirow[t]{2}{*}{113} & \multirow[t]{2}{*}{ ununtrium } & \multirow[t]{2}{*}{ Uut } & 283 & 283.176 & $\sim 0.1$ & $\mathrm{~s}$ \\
\hline & & & 284 & 284.178 & $\sim 0.48$ & $\mathrm{~s}$ \\
\hline \multirow[t]{2}{*}{114} & \multirow[t]{2}{*}{ ununquadium } & \multirow[t]{2}{*}{ Uuq } & 288 & 288.186 & $0.8(3)$ & $\mathrm{s}$ \\
\hline & & & 289 & 289.189 & $\sim 2.6$ & $\mathrm{~s}$ \\
\hline \multirow[t]{2}{*}{115} & \multirow[t]{2}{*}{ ununpentium } & \multirow[t]{2}{*}{ Uup } & 287 & 287.191 & $\sim 0.03$ & $\mathrm{~s}$ \\
\hline & & & 288 & 288.192 & $\sim 0.09$ & s \\
\hline \multirow[t]{3}{*}{116} & \multirow[t]{3}{*}{ ununhexium } & \multirow[t]{3}{*}{ Uuh } & 291 & & $\sim 18 \times 10^{-3}$ & $\mathrm{~s}$ \\
\hline & & & 292 & 292.200 & $\sim 18 \times 10^{-3}$ & $\mathrm{~s}$ \\
\hline & & & 293 & & $0.06(5)$ & $\mathrm{s}$ \\
\hline 118 & ununoctium & Uuo & 294 & & $\sim 0.89 \times 10^{-3}$ & s \\
\hline
\end{tabular}

Table 4 Standard atomic weights 2007 abridged to four significant digits. [Scaled to $A_{\mathrm{r}}\left({ }^{12} \mathrm{C}\right)=12$, where ${ }^{12} \mathrm{C}$ is a neutral atom in its nuclear and electronic ground state.]

The atomic weights of many elements are not invariant, but depend on the origin and treatment of the material. The standard values of $A_{\mathrm{r}}(\mathrm{E})$ and the uncertainties (in parentheses, following the last significant figure to which they are attributed) apply to elements of natural terrestrial origin. The last significant figure of each tabulated value is considered reliable to \pm 1 except when a larger single digit uncertainty is inserted in parentheses following the atomic weight.

\begin{tabular}{lllc}
\hline Atomic number & \multicolumn{1}{c}{ Element name } & Symbol & Atomic weight \\
\hline 1 & hydrogen & $\mathrm{H}$ & 1.008 \\
2 & helium & $\mathrm{He}$ & 4.003 \\
3 & lithium & $\mathrm{Li}$ & $6.941(2)^{\dagger}$ \\
4 & beryllium & $\mathrm{Be}$ & 9.012 \\
5 & boron & $\mathrm{B}$ & $10.81^{\#}$ \\
6 & carbon & $\mathrm{C}$ & 12.01 \\
7 & nitrogen & $\mathrm{N}$ & 14.01 \\
8 & oxygen & $\mathrm{O}$ & 16.00 \\
9 & fluorine & $\mathrm{F}$ & 19.00 \\
10 & neon & $\mathrm{Ne}$ & 20.18 \\
11 & sodium & $\mathrm{Na}$ & 22.99 \\
12 & magnesium & $\mathrm{Mg}$ & 24.31 \\
13 & aluminium (aluminum) & $\mathrm{Al}$ & 26.98 \\
14 & silicon & $\mathrm{Si}$ & 28.09
\end{tabular}


Table 4 (Continued).

\begin{tabular}{|c|c|c|c|}
\hline Atomic number & Element name & Symbol & Atomic weight \\
\hline 15 & phosphorus & $\mathrm{P}$ & 30.97 \\
\hline 16 & sulfur & S & $32.07^{\#}$ \\
\hline 17 & chlorine & $\mathrm{Cl}$ & 35.45 \\
\hline 18 & argon & $\mathrm{Ar}$ & 39.95 \\
\hline 19 & potassium & $\mathrm{K}$ & 39.10 \\
\hline 20 & calcium & $\mathrm{Ca}$ & $40.08^{\#}$ \\
\hline 21 & scandium & $\mathrm{Sc}$ & 44.96 \\
\hline 22 & titanium & $\mathrm{Ti}$ & 47.87 \\
\hline 23 & vanadium & $\mathrm{V}$ & 50.94 \\
\hline 24 & chromium & $\mathrm{Cr}$ & 52.00 \\
\hline 25 & manganese & Mn & 54.94 \\
\hline 26 & iron & $\mathrm{Fe}$ & 55.85 \\
\hline 27 & cobalt & $\mathrm{Co}$ & 58.93 \\
\hline 28 & nickel & $\mathrm{Ni}$ & 58.69 \\
\hline 29 & copper & $\mathrm{Cu}$ & 63.55 \\
\hline 30 & zinc & $\mathrm{Zn}$ & $65.38(2)$ \\
\hline 31 & gallium & $\mathrm{Ga}$ & 69.72 \\
\hline 32 & germanium & $\mathrm{Ge}$ & 72.64 \\
\hline 33 & arsenic & As & 74.92 \\
\hline 34 & selenium & $\mathrm{Se}$ & $78.96(3)$ \\
\hline 35 & bromine & $\mathrm{Br}$ & 79.90 \\
\hline 36 & krypton & $\mathrm{Kr}$ & $83.80^{\#}$ \\
\hline 37 & rubidium & $\mathrm{Rb}$ & $85.47^{\#}$ \\
\hline 38 & strontium & $\mathrm{Sr}$ & $87.61^{\#}$ \\
\hline 39 & yttrium & $\mathrm{Y}$ & 88.91 \\
\hline 40 & zirconium & $\mathrm{Zr}$ & $91.22^{\#}$ \\
\hline 41 & niobium & $\mathrm{Nb}$ & 92.91 \\
\hline 42 & molybdenum & Mo & $95.96(2)^{\#}$ \\
\hline 43 & technetium* & $\mathrm{Tc}$ & \\
\hline 44 & ruthenium & $\mathrm{Ru}$ & $101.1^{\#}$ \\
\hline 45 & rhodium & $\mathrm{Rh}$ & 102.9 \\
\hline 46 & palladium & $\mathrm{Pd}$ & $106.4^{\#}$ \\
\hline 47 & silver & $\mathrm{Ag}$ & $107.9^{\#}$ \\
\hline 48 & cadmium & $\mathrm{Cd}$ & $112.4^{\#}$ \\
\hline 49 & indium & In & 114.8 \\
\hline 50 & tin & $\mathrm{Sn}$ & $118.7^{\#}$ \\
\hline 51 & antimony & $\mathrm{Sb}$ & $121.8^{\#}$ \\
\hline 52 & tellurium & $\mathrm{Te}$ & $127.6^{\#}$ \\
\hline 53 & iodine & I & 126.9 \\
\hline 54 & xenon & $\mathrm{Xe}$ & $131.3^{\#}$ \\
\hline 55 & caesium (cesium) & $\mathrm{Cs}$ & 132.9 \\
\hline 56 & barium & $\mathrm{Ba}$ & 137.3 \\
\hline 57 & lanthanum & $\mathrm{La}$ & 138.9 \\
\hline 58 & cerium & $\mathrm{Ce}$ & $140.1^{\#}$ \\
\hline 59 & praseodymium & $\operatorname{Pr}$ & 140.9 \\
\hline 60 & neodymium & $\mathrm{Nd}$ & $144.2^{\#}$ \\
\hline 61 & promethium* & $\mathrm{Pm}$ & \\
\hline 62 & samarium & $\mathrm{Sm}$ & $150.4^{\#}$ \\
\hline 63 & europium & $\mathrm{Eu}$ & $152.0^{\#}$ \\
\hline
\end{tabular}

(continues on next page) 
Table 4 (Continued).

\begin{tabular}{|c|c|c|c|}
\hline Atomic number & Element name & Symbol & Atomic weight \\
\hline 64 & gadolinium & $\mathrm{Gd}$ & $157.3^{\#}$ \\
\hline 65 & terbium & $\mathrm{Tb}$ & 158.9 \\
\hline 66 & dysprosium & Dy & $162.5^{\#}$ \\
\hline 67 & holmium & Ho & 164.9 \\
\hline 68 & erbium & $\mathrm{Er}$ & $167.3^{\#}$ \\
\hline 69 & thulium & $\mathrm{Tm}$ & 168.9 \\
\hline 70 & ytterbium & $\mathrm{Yb}$ & $173.1^{\#}$ \\
\hline 71 & lutetium & $\mathrm{Lu}$ & 175.0 \\
\hline 72 & hafnium & Hf & 178.5 \\
\hline 73 & tantalum & $\mathrm{Ta}$ & 180.9 \\
\hline 74 & tungsten & $\mathrm{W}$ & 183.9 \\
\hline 75 & rhenium & $\operatorname{Re}$ & 186.2 \\
\hline 76 & osmium & Os & 190.2 \\
\hline 77 & iridium & Ir & 192.2 \\
\hline 78 & platinum & $\mathrm{Pt}$ & 195.1 \\
\hline 79 & gold & $\mathrm{Au}$ & 197.0 \\
\hline 80 & mercury & $\mathrm{Hg}$ & 200.6 \\
\hline 81 & thallium & $\mathrm{Tl}$ & 204.4 \\
\hline 82 & lead & $\mathrm{Pb}$ & $207.2^{\#}$ \\
\hline 83 & bismuth & $\mathrm{Bi}$ & 209.0 \\
\hline 84 & polonium* & Po & \\
\hline 85 & astatine* & At & \\
\hline 86 & radon* & $\mathrm{Rn}$ & \\
\hline 87 & francium* & $\mathrm{Fr}$ & \\
\hline 88 & radium* & $\mathrm{Ra}$ & \\
\hline 89 & actinium* & $\mathrm{Ac}$ & \\
\hline 90 & thorium* & Th & 232.0 \\
\hline 91 & protactinium* & $\mathrm{Pa}$ & 231.0 \\
\hline 92 & uranium* & $\mathrm{U}$ & $238.0^{\#}$ \\
\hline 93 & neptunium* & $\mathrm{Np}$ & \\
\hline 94 & plutonium* & $\mathrm{Pu}$ & \\
\hline 95 & americium* & $\mathrm{Am}$ & \\
\hline 96 & curium* & $\mathrm{Cm}$ & \\
\hline 97 & berkelium* & $\mathrm{Bk}$ & \\
\hline 98 & californium* & $\mathrm{Cf}$ & \\
\hline 99 & einsteinium* & Es & \\
\hline 100 & fermium* & $\mathrm{Fm}$ & \\
\hline 101 & mendelevium* & $\mathrm{Md}$ & \\
\hline 102 & nobelium* & No & \\
\hline 103 & lawrencium* & $\mathrm{Lr}$ & \\
\hline 104 & rutherfordium* & $\mathrm{Rf}$ & \\
\hline 105 & dubnium* & $\mathrm{Db}$ & \\
\hline 106 & seaborgium* & $\mathrm{Sg}$ & \\
\hline 107 & bohrium* & $\mathrm{Bh}$ & \\
\hline 108 & hassium* & Hs & \\
\hline 109 & meitnerium* & Mt & \\
\hline 110 & darmstadtium* & Ds & \\
\hline 111 & roentgenium* & $\mathrm{Rg}$ & \\
\hline 112 & ununbium* & Uub & \\
\hline
\end{tabular}


Table 4 (Continued).

\begin{tabular}{llll}
\hline Atomic number & Element name & Symbol & Atomic weight \\
\hline 113 & ununtrium* & Uut & \\
114 & ununquadium* & Uuq & \\
115 & ununpentium* & Uup & \\
116 & ununhexium* & Uuh & \\
118 & ununoctium* & Uuo & \\
\hline
\end{tabular}

*Element has no stable nuclides. One or more well-known isotopes are given in Table 3 with the appropriate relative atomic mass and half-life. However, three such elements ( Th, $\mathrm{Pa}$, and $\mathrm{U}$ ) do have a characteristic terrestrial isotopic composition, and for these an atomic weight is tabulated.

Commercially available $\mathrm{Li}$ materials have atomic weights that range between 6.939 and 6.996; if a more accurate value is required, it must be determined for the specific material.

\#Values may differ from the atomic weights of the relevant elements in some naturally occurring samples because of a variation in the relative isotopic abundance.

Table 5 Standard atomic weights 2007 abridged to five significant digits. [Scaled to $\left.A_{\mathrm{r}}{ }^{12} \mathrm{C}\right)=12$, where ${ }^{12} \mathrm{C}$ is a neutral atom in its nuclear and electronic ground state.]

Atomic weights are quoted here to five significant figures unless the dependable accuracy is further limited by either the combined uncertainties of the best published atomic weight determinations, or by the variability of isotopic composition in normal terrestrial occurrences (the latter applies to the elements annotated "r"). The last significant figure of each tabulated value is considered reliable to \pm 1 except when a larger single digit uncertainty is inserted in parentheses following the atomic weight. Neither the highest nor the lowest actual atomic weight of any normal sample is thought likely to differ from the tabulated values by more than one assigned uncertainty. However, the tabulated values do not apply either to samples of highly exceptional isotopic composition arising from most unusual geological occurrences (for elements annotated "g") or to those whose isotopic composition has been artificially altered. Such might even be found in commerce without disclosure of that modification (for elements annotated " $m$ "). Elements with no stable isotope do not have an atomic weight and such entries have a blank in the atomic weight column. However, three such elements ( $\mathrm{Th}, \mathrm{Pa}$, and $\mathrm{U}$ ) do have a characteristic terrestrial isotopic composition and for these an atomic weight value is tabulated. For more detailed information, users should refer to the full IUPAC Table of Standard Atomic Weights. Names of elements with atomic number 112 to 118 are provisional.

\begin{tabular}{lllcc}
\hline \multicolumn{5}{c}{ Order of atomic number } \\
\hline Atomic number & \multicolumn{1}{c}{ Element name } & Symbol & Atomic weight & Footnotes \\
\hline 1 & hydrogen & $\mathrm{H}$ & 1.0079 & $\mathrm{~g} \mathrm{~m}$ \\
2 & helium & $\mathrm{He}$ & 4.0026 & \\
3 & lithium & $\mathrm{Li}$ & $6.941(2)^{\dagger}$ & $\mathrm{g} \mathrm{m} \mathrm{r}$ \\
4 & beryllium & $\mathrm{Be}$ & 9.0122 & \\
5 & boron & $\mathrm{B}$ & $10.811(7)$ & $\mathrm{g} \mathrm{m} \mathrm{r}$ \\
6 & carbon & $\mathrm{C}$ & 12.011 & $\mathrm{~g}$ \\
7 & nitrogen & $\mathrm{N}$ & 14.007 & \\
8 & oxygen & $\mathrm{O}$ & 15.999 & \\
9 & fluorine & $\mathrm{F}$ & 18.998 & $\mathrm{~m}$ \\
10 & neon & $\mathrm{Ne}$ & 20.180 &
\end{tabular}

(continues on next page) 
Table 5 (Continued).

\begin{tabular}{|c|c|c|c|c|c|}
\hline \multicolumn{6}{|c|}{ Order of atomic number } \\
\hline Atomic number & Element name & Symbol & Atomic weight & Foot & notes \\
\hline 11 & sodium & $\mathrm{Na}$ & 22.990 & & \\
\hline 12 & magnesium & $\mathrm{Mg}$ & 24.305 & & \\
\hline 13 & aluminium (aluminum) & $\mathrm{Al}$ & 26.982 & & \\
\hline 14 & silicon & $\mathrm{Si}$ & 28.086 & & \\
\hline 15 & phosphorus & $\mathrm{P}$ & 30.974 & & \\
\hline 16 & sulfur & $\mathrm{S}$ & $32.065(5)$ & $\mathrm{g}$ & $\mathrm{r}$ \\
\hline 17 & chlorine & $\mathrm{Cl}$ & $35.453(2)$ & 1 & $\mathrm{n}$ \\
\hline 18 & argon & $\mathrm{Ar}$ & 39.948 & $\mathrm{~g}$ & $\mathrm{r}$ \\
\hline 19 & potassium & $\mathrm{K}$ & 39.098 & $\mathrm{~g}$ & \\
\hline 20 & calcium & $\mathrm{Ca}$ & $40.078(4)$ & $\mathrm{g}$ & \\
\hline 21 & scandium & $\mathrm{Sc}$ & 44.956 & & \\
\hline 22 & titanium & $\mathrm{Ti}$ & 47.867 & & \\
\hline 23 & vanadium & $\mathrm{V}$ & 50.942 & & \\
\hline 24 & chromium & $\mathrm{Cr}$ & 51.996 & & \\
\hline 25 & manganese & $\mathrm{Mn}$ & 54.938 & & \\
\hline 26 & iron & $\mathrm{Fe}$ & $55.845(2)$ & & \\
\hline 27 & cobalt & Co & 58.933 & & \\
\hline 28 & nickel & $\mathrm{Ni}$ & 58.693 & & $\mathrm{r}$ \\
\hline 29 & copper & $\mathrm{Cu}$ & $63.546(3)$ & & $\mathrm{r}$ \\
\hline 30 & zinc & $\mathrm{Zn}$ & $65.38(2)$ & & $\mathrm{r}$ \\
\hline 31 & gallium & $\mathrm{Ga}$ & 69.723 & & \\
\hline 32 & germanium & $\mathrm{Ge}$ & 72.64 & & \\
\hline 33 & arsenic & As & 74.922 & & \\
\hline 34 & selenium & $\mathrm{Se}$ & $78.96(3)$ & & $\mathrm{r}$ \\
\hline 35 & bromine & $\mathrm{Br}$ & 79.904 & & \\
\hline 36 & krypton & $\mathrm{Kr}$ & $83.798(2)$ & $g_{1}$ & \\
\hline 37 & rubidium & $\mathrm{Rb}$ & 85.468 & & \\
\hline 38 & strontium & $\mathrm{Sr}$ & 87.62 & $\mathrm{~g}$ & $\mathrm{r}$ \\
\hline 39 & yttrium & $\mathrm{Y}$ & 88.906 & & \\
\hline 40 & zirconium & $\mathrm{Zr}$ & $91.224(2)$ & $\mathrm{g}$ & \\
\hline 41 & niobium & $\mathrm{Nb}$ & $92.906(2)$ & & \\
\hline 42 & molybdenum & Mo & $95.96(2)$ & $\mathrm{g}$ & $\mathrm{r}$ \\
\hline 43 & technetium* & $\mathrm{Tc}$ & & & \\
\hline 44 & ruthenium & $\mathrm{Ru}$ & $101.07(2)$ & $\mathrm{g}$ & \\
\hline 45 & rhodium & $\mathrm{Rh}$ & 102.91 & & \\
\hline 46 & palladium & $\mathrm{Pd}$ & 106.42 & $\mathrm{~g}$ & \\
\hline 47 & silver & $\mathrm{Ag}$ & 107.87 & $\mathrm{~g}$ & \\
\hline 48 & cadmium & $\mathrm{Cd}$ & 112.41 & & \\
\hline 49 & indium & In & 114.82 & & \\
\hline 50 & $\operatorname{tin}$ & $\mathrm{Sn}$ & 118.71 & & \\
\hline 51 & antimony & $\mathrm{Sb}$ & 121.76 & $\mathrm{~g}$ & \\
\hline 52 & tellurium & $\mathrm{Te}$ & $127.60(3)$ & $\mathrm{g}$ & \\
\hline 53 & iodine & I & 126.90 & & \\
\hline 54 & xenon & $\mathrm{Xe}$ & 131.29 & $g_{1}$ & \\
\hline 55 & caesium (cesium) & $\mathrm{Cs}$ & 132.91 & & \\
\hline 56 & barium & $\mathrm{Ba}$ & 137.33 & & \\
\hline 57 & lanthanum & $\mathrm{La}$ & 138.91 & & \\
\hline 58 & cerium & $\mathrm{Ce}$ & 140.12 & $\mathrm{~g}$ & \\
\hline
\end{tabular}


Table 5 (Continued).

\begin{tabular}{|c|c|c|c|c|}
\hline \multicolumn{5}{|c|}{ Order of atomic number } \\
\hline Atomic number & Element name & Symbol & Atomic weight & Footnotes \\
\hline 59 & praseodymium & $\operatorname{Pr}$ & 140.91 & \\
\hline 60 & neodymium & $\mathrm{Nd}$ & 144.24 & g \\
\hline 61 & promethium* & $\mathrm{Pm}$ & & \\
\hline 62 & samarium & $\mathrm{Sm}$ & $150.36(2)$ & $\mathrm{g}$ \\
\hline 63 & europium & $\mathrm{Eu}$ & 151.96 & $\mathrm{~g}$ \\
\hline 64 & gadolinium & Gd & $157.25(3)$ & $\mathrm{g}$ \\
\hline 65 & terbium & $\mathrm{Tb}$ & 158.93 & \\
\hline 66 & dysprosium & Dy & 162.50 & $\mathrm{~g}$ \\
\hline 67 & holmium & Ho & 164.93 & \\
\hline 68 & erbium & $\mathrm{Er}$ & 167.26 & $\mathrm{~g}$ \\
\hline 69 & thulium & $\mathrm{Tm}$ & 168.93 & \\
\hline 70 & ytterbium & $\mathrm{Yb}$ & 173.05 & $\mathrm{~g}$ \\
\hline 71 & lutetium & $\mathrm{Lu}$ & 174.97 & $\mathrm{~g}$ \\
\hline 72 & hafnium & Hf & $178.49(2)$ & \\
\hline 73 & tantalum & $\mathrm{Ta}$ & 180.95 & \\
\hline 74 & tungsten & $\mathrm{W}$ & 183.84 & \\
\hline 75 & rhenium & $\mathrm{Re}$ & 186.21 & \\
\hline 76 & osmium & Os & $190.23(3)$ & $\mathrm{g}$ \\
\hline 77 & iridium & $\mathrm{Ir}$ & 192.22 & \\
\hline 78 & platinum & $\mathrm{Pt}$ & 195.08 & \\
\hline 79 & gold & $\mathrm{Au}$ & 196.97 & \\
\hline 80 & mercury & $\mathrm{Hg}$ & $200.59(2)$ & \\
\hline 81 & thallium & $\mathrm{Tl}$ & 204.38 & \\
\hline 82 & lead & $\mathrm{Pb}$ & 207.2 & $\begin{array}{ll}\mathrm{g} & \mathrm{r}\end{array}$ \\
\hline 83 & bismuth & $\mathrm{Bi}$ & 208.98 & \\
\hline 84 & polonium* & Po & & \\
\hline 85 & astatine* & At & & \\
\hline 86 & radon* & $\mathrm{Rn}$ & & \\
\hline 87 & francium* & $\mathrm{Fr}$ & & \\
\hline 88 & radium* & $\mathrm{Ra}$ & & \\
\hline 89 & actinium* & Ac & & \\
\hline 90 & thorium* & $\mathrm{Th}$ & 232.04 & $\mathrm{~g}$ \\
\hline 91 & protactinium* & $\mathrm{Pa}$ & 231.04 & \\
\hline 92 & uranium* & $\mathrm{U}$ & 238.03 & $\mathrm{~g} \mathrm{~m}$ \\
\hline 93 & neptunium* & $\mathrm{Np}$ & & \\
\hline 94 & plutonium* & $\mathrm{Pu}$ & & \\
\hline 95 & americium* & Am & & \\
\hline 96 & curium* & $\mathrm{Cm}$ & & \\
\hline 97 & berkelium* & $\mathrm{Bk}$ & & \\
\hline 98 & californium* & $\mathrm{Cf}$ & & \\
\hline 99 & einsteinium* & Es & & \\
\hline 100 & fermium* & $\mathrm{Fm}$ & & \\
\hline 101 & mendelevium* & $\mathrm{Md}$ & & \\
\hline 102 & nobelium* & No & & \\
\hline 103 & lawrencium* & $\mathrm{Lr}$ & & \\
\hline 104 & rutherfordium* & $\mathrm{Rf}$ & & \\
\hline 105 & dubnium* & $\mathrm{Db}$ & & \\
\hline 106 & seaborgium* & $\mathrm{Sg}$ & & \\
\hline
\end{tabular}

(continues on next page) 
Table 5 (Continued).

\begin{tabular}{lllll}
\hline \multicolumn{4}{c}{ Order of atomic number } & \\
\hline Atomic number & \multicolumn{1}{c}{ Element name } & Symbol & Atomic weight & Footnotes \\
\hline 107 & bohrium* & $\mathrm{Bh}$ & \\
108 & hassium* & $\mathrm{Hs}$ & \\
109 & meitnerium* & $\mathrm{Mt}$ & \\
110 & darmstadtium* & $\mathrm{Ds}$ & \\
111 & roentgenium* & $\mathrm{Rg}$ & \\
112 & ununbium* & Uub & \\
113 & ununtrium* & Uut & \\
114 & ununquadium* & Uuq & \\
115 & ununpentium* & Uup & \\
116 & ununhexium* & Uuh & \\
118 & ununoctium* & Uuo & \\
\hline
\end{tabular}

*Element has no stable nuclides. One or more well-known isotopes are given in Table 3 with the appropriate relative atomic mass and half-life. However, three such elements (Th, $\mathrm{Pa}$, and $\mathrm{U}$ ) do have a characteristic terrestrial isotopic composition, and for these an atomic weight is tabulated.

${ }^{\dagger}$ Commercially available Li materials have atomic weights that range between 6.939 and 6.996; if a more accurate value is required, it must be determined for the specific material.

g Geological specimens are known in which the element has an isotopic composition outside the limits for normal material. The difference between the atomic weight of the element in such specimens and that given in the table may exceed the stated uncertainty.

m Modified isotopic compositions may be found in commercially available material because it has been subjected to an undisclosed or inadvertent isotopic fractionation. Substantial deviations in atomic weight of the element from that given in the table can occur.

$\mathrm{r} \quad$ Range in isotopic composition of normal terrestrial material prevents a more precise $A_{\mathrm{r}}(\mathrm{E})$ being given; the tabulated $A_{\mathrm{r}}(\mathrm{E})$ value and uncertainty should be applicable to normal material.

\section{COMMENTS ON EVALUATIONS OF ATOMIC WEIGHTS AND ANNOTATIONS}

The Commission regularly evaluates reports of atomic weight determinations to select the "best measurement" of the isotopic abundances of an element in a specified material. The best measurement may be defined as a set of analyses of the isotope-amount or isotope-number ratios of an element in a wellcharacterized, representative material with low combined uncertainty. To be considered by the Commission for evaluation, reports must be published in peer-reviewed literature, and the results should be given with sufficient detail that the Commission can reconstruct the uncertainty budget in its various components, including sample preparation, analysis of isotope-amount or isotope-number ratios, and data handling.

Criteria used to evaluate a "best measurement" include:

1. The extent to which random and systematic errors have been assessed and documented in the report. The Commission seeks evidence that mass spectrometer linearity, mass spectrometric fractionation of ions of varying masses, memory, baseline, interference between ions, sample purity and preparation effects, and statistical assessment of data were carried out properly. Preference is given to measurements that are fully calibrated with synthetic mixtures of isotopes of the element of interest, covering the isotopic abundance variations of naturally occurring materials over the range of the masses of the isotopes in the material being analyzed.

2. The relevance and availability of the analyzed material for the scientific community involved in isotopic measurements and calibrations. Preference is given to analyses of chemically stable materials that are distributed internationally as isotopic reference materials, e.g., by the U.S. National Institute of Standards and Technology (NIST), the European Institute of Reference Materials and 
Measurements (IRMM), the International Atomic Energy Agency (IAEA), etc. or to isotopically unfractionated representatives of homogeneous terrestrial materials.

New analytical techniques for isotope-amount ratio determinations have made a significant impact on how data are collected and reported. In the case of $\mathrm{Zn}$, for the first time in the history of the Commission, a standard atomic weight was recommended based on a new best measurement determined by multiple collector inductively coupled plasma mass spectrometry (MC-ICP-MS).

Following are brief descriptions of the changes in the Table of Standard Atomic Weights resulting from the Commission meeting in 2007.

\section{Lutetium}

The Commission has changed the recommended value for the standard atomic weight of lutetium, $A_{\mathrm{r}}(\mathrm{Lu})$, to $174.9668(1)$, based on a recent measurement by [10]. The measurement does not qualify for a fully calibrated measurement because the linearity of the instrument was verified for a certified potassium isotopic reference material (NIST 985), but not $\mathrm{Lu}$. $\mathrm{Sr}$ and $\mathrm{Pb}$ isotopic compositions were also employed to check for linearity. The instrumental isotopic fractionation of Lu was corrected using a fractionation factor determined for $\mathrm{Yb}$ [11]. The relatively low abundance of the ${ }^{176} \mathrm{Lu}$ in this two-isotope element means that even when applying the Commission's standard uncertainty protocol of increasing the reported uncertainty by $100 \%$ because of an uncalibrated fractionation factor, the corrections still result in a standard atomic weight uncertainty reduction of a factor of 10 over previous determined values. The ${ }^{176} \mathrm{Lu} /{ }^{175} \mathrm{Lu}$ isotope-amount ratio, $n\left({ }^{176} \mathrm{Lu}\right) / n\left({ }^{175} \mathrm{Lu}\right)$, reported by [10] is also determined by the Commission to be the best measurement from a single terrestrial source and the isotope amount abundances are $x\left({ }^{175} \mathrm{Lu}\right)=0.974013(12)$ and $x\left({ }^{176} \mathrm{Lu}\right)=0.025987(12)$. The previous atomic weight value $A_{\mathrm{r}}(\mathrm{Lu})=174.967(1)$, recommended in 1981, was based on the mass spectrometric measurements of McCulloch et al. [12]. Historical values of $A_{\mathrm{r}}(\mathrm{Lu})$ include [8]: 1909, 174.0; 1916, 175.0; 1940, $174.99 ; 1961,174.97 ; 1969,174.97(1) ; 1977,174.967(3) ; 1981,174.967(1)$.

\section{Molybdenum}

The Commission has changed the recommended value for the standard atomic weight of molybdenum, $A_{\mathrm{r}}(\mathrm{Mo})$, to $95.96(2)$, based on the first calibrated measurements with synthetic isotope mixtures using a thermal ionization mass spectrometer (TIMS) [13]. Although the authors of ref. [13] reduced uncertainties with renormalization of the ratios using the calculated fractionation factors of $N\left({ }^{100} \mathrm{Mo}\right) / N\left({ }^{95} \mathrm{Mo}\right)$, the Commission adopted the conventional method of uncertainty calculations using the raw data and assigned the uncertainty of 0.02 . The new value agrees marginally with the previous value of $A_{\mathrm{r}}(\mathrm{Mo})$ of 95.94(2) with its enlarged uncertainty recommended in 2001 [14]. The new calibrated values for the isotope amount abundances of Mo determined by the Commission to be the best measurement from a single terrestrial source are $x\left({ }^{92} \mathrm{Mo}\right)=0.14525(15), x\left({ }^{94} \mathrm{Mo}\right)=0.091514(74)$, $x\left({ }^{95} \mathrm{Mo}\right)=0.158375(98), x\left({ }^{96} \mathrm{Mo}\right)=0.16672(19), x\left({ }^{97} \mathrm{Mo}\right)=0.095991(73), x\left({ }^{98} \mathrm{Mo}\right)=0.24391(18)$, and $x\left({ }^{100} \mathrm{Mo}\right)=0.09824(50)$. The previous value of $A_{\mathrm{r}}(\mathrm{Mo})$ was based on chemical ratio measurements [15], and the evaluated uncertainty of 0.03 was later included in 1969. The Commission reduced the uncertainty to 0.01 in 1975 but reevaluated and changed it to 0.02 in 2001 . Historical values of $A_{\mathrm{r}}(\mathrm{Mo})$ include [8]: 1882, 95.75; 1894, 96; 1896, 95.98; 1897, 95.99; 1900, 96; 1938, 95.95; 1961, 95.94; 1969, 95.94(3); 1975, 95.94(1); 2001, 95.94(2).

\section{Nickel}

The Commission has changed the recommended value for the standard atomic weight of nickel, $A_{\mathrm{r}}(\mathrm{Ni})$, to 58.6934(4), based on the measurements published in [16]. The authors measured $\mathrm{Ni}$ isotope-amount 
ratios on an MC-ICP-MS with external normalization using doped Cu (SRM 976). They found that the atomic weights of a Ni reagent and nickel sulfide ore were outside of the range of values in the previous Table of Standard Atomic Weights. The footnotes " $r$ " in Tables 1, 2, and 5 arise because the variation in the atomic weights of $\mathrm{Ni}$ from normal sources limit the precision to which $A_{\mathrm{r}}(\mathrm{Ni})$ may be reported. Historical values of $A_{\mathrm{r}}(\mathrm{Ni})$ include [8]: 1892, 58.06; 1894, 58.7; 1896, 58.69; 1900, 58.7; 1909, 58.68; 1925, 58.69; 1955, 58.71; 1969, 58.71(3); 1973, 58.70(1); 1979, 58.69(1); 1989, 58.693 4(2).

\section{Ytterbium}

The Commission has changed the recommended value for the standard atomic weight of ytterbium, $A_{\mathrm{r}}(\mathrm{Yb})$, to $173.054(5)$, based on the first calibrated measurements with synthetic isotope mixtures using a TIMS [11]. The isotopic composition of $\mathrm{Yb}$ reported in [11] is also determined by the Commission to be the best measurement from a single terrestrial source. The isotope amount abundances are $x\left({ }^{168} \mathrm{Yb}\right)=0.001232(4), x\left({ }^{170} \mathrm{Yb}\right)=0.02982(6), x\left({ }^{171} \mathrm{Yb}\right)=0.14086(20), x\left({ }^{172} \mathrm{Yb}\right)=0.21686(19)$, $x\left({ }^{173} \mathrm{Yb}\right)=0.16103(9), x\left({ }^{174} \mathrm{Yb}\right)=0.32025(12), x\left({ }^{176} \mathrm{Yb}\right)=0.12995(13)$. The previous value of $A_{\mathrm{r}}(\mathrm{Yb})$ of 173.04(3) was originally based on the chemical determination from 1934 and was confirmed by mass spectrometric data in [17,18] as summarized by the Commission in 1961 [19]. The evaluated uncertainty was added for the first time in 1969. Historical values of $A_{\mathrm{r}}(\mathrm{Yb})$ include [8]: 1882, 173.16; 1894, 173.0; 1897, 173.19; 1900, 173.2; 1903, 173; 1909, 172.0; 1916, 173.5; 1925, 173.6; 1931, 173.5; 1934, $173.04 ; 1969,173.04(3)$.

\section{Zinc}

The Commission has changed the recommended value for the standard atomic weight of zinc, $A_{r}(\mathrm{Zn})$, to 65.38(2) based on a fully calibrated measurement [20]. The authors calibrated their instrument using synthetic mixtures of highly enriched $\mathrm{Zn}$ isotopes. Their value for $A_{\mathrm{r}}(\mathrm{Zn})$ measured from the isotope reference material IRMM-3702 is $65.37777(22)$, which is significantly different from the previous IUPAC value of 65.409(4) [1,2]. The latter is based on a calibrated measurement by Chang et al. using TIMS [21]. A calibrated MC-ICP-MS measurement by Tanimizu et al. [22] found an atomic weight of $\mathrm{Zn}$ of 65.3756(40), also significantly different from the earlier value [21]. It is important to note that the atomic weight of $\mathrm{Zn}$ of 65.377(3) determined much earlier using coulometric techniques [23] is in good agreement with the measurement by Ponzevera et al. [20] and Tanimizu et al. [22]. Both [20,22] proposed that the atomic weight reported in Chang et al. [21] was affected by a systematic measurement bias. Ponzevera et al. analyzed an aliquot of the same material as Chang et al. (IM-1009 in ref. [20] and Sample 2 in ref. [21]) and found a significant difference between their MC-ICP-MS result and the value reported in Chang et al. [21].

Ponzevera et al. [20] also analyzed a commercial Zn shelf reagent (IRM-651) for its absolute isotopic composition. This measurement confirms earlier reports in the literature of significant variations in $\mathrm{Zn}$ isotopic composition in natural geological samples and $\mathrm{Zn}$ reagents. The standard atomic weight of $\mathrm{Zn}$ and its associated uncertainty recommended by the Commission 65.38(2) includes the reported variations in natural $\mathrm{Zn}$ isotopic abundances and, thus, variations in the $\mathrm{Zn}$ atomic weight in nature. This recommendation also includes the $\mathrm{Zn}$ atomic weight of 65.396(5), which was obtained by a calibrated measurement of an unspecified $\mathrm{Zn}$ sample by electron impact mass spectrometry [24]. The uncertainty reported in [20] was expanded to encompass known variations in the isotopic composition of $\mathrm{Zn}$ in natural materials. The footnotes " $r$ " in Tables 1, 2, and 5 arise because the variations in the atomic weights of $\mathrm{Zn}$ from normal sources limits the precision to which $A_{\mathrm{r}}(\mathrm{Zn})$ may be reported. The isotope amount abundances of IRMM-3702 reported by [20] were also determined by the Commission to be the best measurement from a single terrestrial source and the isotope amount abundances are $x\left({ }^{64} \mathrm{Zn}\right)=$ $0.491704(83), x\left({ }^{66} \mathrm{Zn}\right)=0.27731(11), x\left({ }^{67} \mathrm{Zn}\right)=0.040401(18), x\left({ }^{68} \mathrm{Zn}\right)=0.184483(69)$, and $x\left({ }^{70} \mathrm{Zn}\right)=$ 
$0.006106(11)$. Historical values of $A_{\mathrm{r}}(\mathrm{Zn})$ include [8]: 1882, 65.05; 1894, 65.3; 1896, 65.41; 1900, $65.4 ; 1909,65.7 ; 1910,65.37 ; 1925$, 65.38; 1961, 65.37; 1969, 65.37(3); 1971, 65.38(1); 1983, 65.39(2); 2005, 65.409(4).

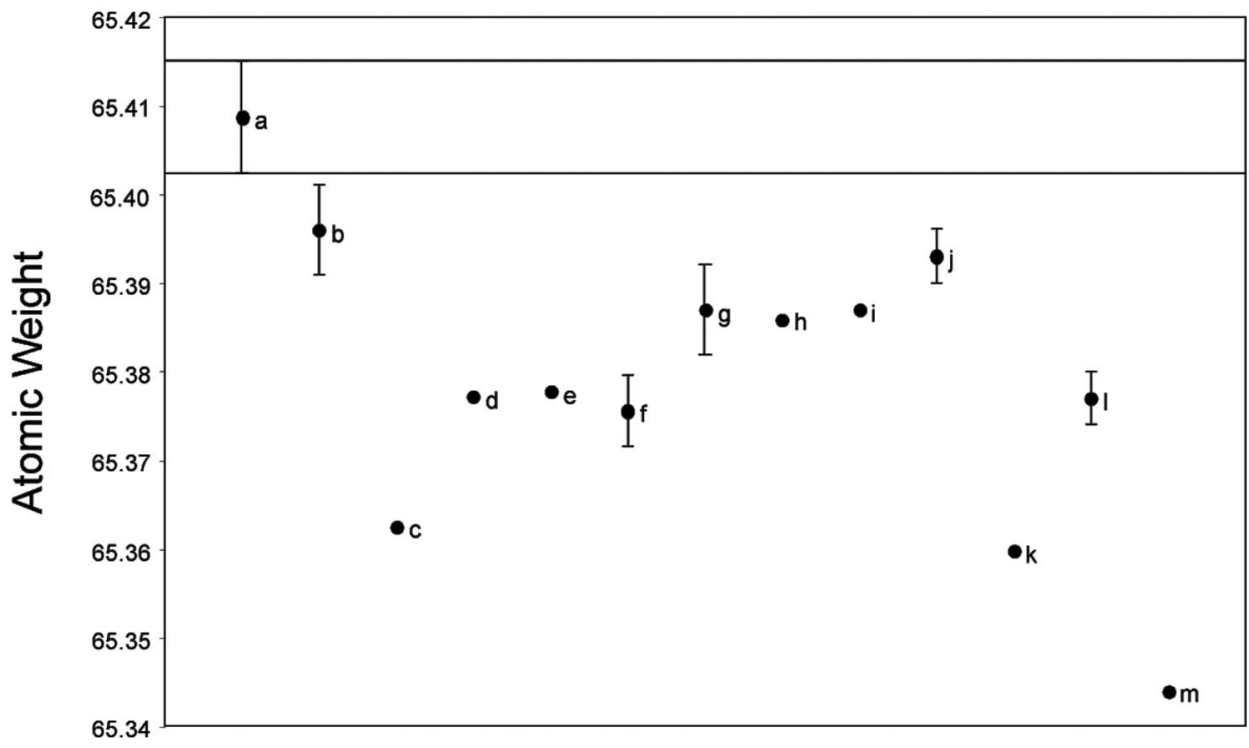

Fig. 1 Atomic weight of $\mathrm{Zn}$ reported in the literature. The values for $A_{\mathrm{r}}(\mathrm{Zn})$ measured over the past six decades do not always agree nor fall within the range recommended previously by IUPAC. The new recommended value for $A_{\mathrm{r}}(\mathrm{Zn})$ now covers most of the atomic weights of $\mathrm{Zn}$ measured in various materials. The footnote " $\mathrm{r}$ " in Tables 1 , 2 , and 5 indicates that the precision in the value of $A_{\mathrm{r}}(\mathrm{Zn})$ is limited by the variability in the isotopic composition measured in normally occurring materials. The data are taken from (a) Chang et al. [21] and IUPAC [1,2], (b) Rosman [24], (c) IRMM-651 [20], (d) IM-1009 [20], (e) IRMM-3702 [20], (f) Tanimizu et al. [22], (g) Leyland et al. [25], (h) Hess et al. [26], (i) Bainbridge et al. [27], (j) Hibbs [28], (k) Okamoto et al. [29], (1) Marinenko et al. [23], and (m) Konishi et al. [30]. The figure is modified from [20].

\section{Relative atomic mass values and half-lives of selected radionuclides}

For elements that have no stable or long-lived nuclides, the data on radioactive half-lives and relative atomic mass values for the nuclides of interest and importance have been evaluated, and the recommended values and uncertainties are listed in Table 3. It must be noted that the listing of particular nuclides for elements numbered 112 and above in Table 3 does not imply any priority of the discovery of those elements on the part of the Commission.

As has been the custom in the past, the Commission publishes a table of relative atomic mass values and half-lives of selected radionuclides, although the Commission has no official responsibility for the dissemination of such values. There is no general agreement on which of the nuclides of the radioactive elements is, or is likely to be judged, "important". Various criteria such as "longest half-life", "production in quantity", and "used commercially" have been applied in the past to the Commission's choice.

The information contained in this table will enable the user to calculate the atomic weights of radioactive materials with a variety of isotopic compositions. Nuclidic mass values have been taken from the 2003 atomic mass Table [6]. Some of these half-lives have already been documented [31-34]. 


\section{ABRIDGED TABLES OF STANDARD ATOMIC WEIGHTS}

It has been noted that the detail and the number of significant figures found in the full Table of Standard Atomic Weights (Tables 1 and 2) exceed the needs and the interests of many users, who are more concerned with the length of time during which a given table has validity to the precision limit of their interests. Tables abridged to four or five significant figures are published with the reasonable hope that not even one of the quoted values will need to be changed because of the biennial revision of the unabridged table, although the quoted uncertainties may be altered. Any change in an abridged value will likely be by only one unit in the final significant figure or by the addition of a fifth significant figure. Such constancy in these values is desirable for textbooks and numerical tables derived from atomic weight data. Standard atomic weights abridged to four and five significant figures are presented in Tables 4 and 5, respectively.

\section{RECOMMENDATION ON THE USE OF OXYGEN IN AIR AS AN INTERNATIONAL REFERENCE STANDARD FOR REPORTING $\delta\left({ }^{17} 0\right)$ AND $\delta\left({ }^{18} 0\right)$ DATA}

Following a proposal by Barkan and Luz [35] and acknowledging the isotopic homogeneity of atmospheric $\mathrm{O}_{2}$, the Commission recommends that atmospheric $\mathrm{O}_{2}$ be recognized as an international measurement standard for measurements of differences in relative $\mathrm{O}$ isotope-amount ratios. The relation for $\delta\left({ }^{17} \mathrm{O}\right)$ is

$$
\delta\left({ }^{17} \mathrm{O}\right)_{\mathrm{B}, \mathrm{Air}}=\frac{n\left({ }^{17} \mathrm{O}\right)_{\mathrm{B}} / n\left({ }^{16} \mathrm{O}\right)_{\mathrm{B}}-n\left({ }^{17} \mathrm{O}\right)_{\mathrm{Air}} / n\left({ }^{16} \mathrm{O}\right)_{\mathrm{Air}}}{n\left({ }^{17} \mathrm{O}\right)_{\mathrm{Air}} / n\left({ }^{16} \mathrm{O}\right)_{\mathrm{Air}}}=\frac{r\left({ }^{17} \mathrm{O}\right)_{\mathrm{B}}-r\left({ }^{17} \mathrm{O}\right)_{\mathrm{Air}}}{r\left({ }^{17} \mathrm{O}\right)_{\mathrm{Air}}}
$$

where $n\left({ }^{17} \mathrm{O}\right)_{\mathrm{B}} / n\left({ }^{16} \mathrm{O}\right)_{\mathrm{B}}$ and $n\left({ }^{17} \mathrm{O}\right)_{\mathrm{Air}} / n\left({ }^{16} \mathrm{O}\right)_{\mathrm{Air}}$ are the ratios of the isotope amounts of specimen $\mathrm{B}$ and atmospheric $\mathrm{O}_{2}$, and $r\left({ }^{17} \mathrm{O}\right)_{\mathrm{B}}$ and $r\left({ }^{16} \mathrm{O}\right)_{\text {Air }}$ are the isotope-amount ratios of specimen $\mathrm{B}$ and atmospheric $\mathrm{O}_{2}$. In an equivalent manner, the relation for $\delta\left({ }^{18} \mathrm{O}\right)$ is

$$
\delta\left({ }^{18} \mathrm{O}\right)_{\mathrm{B}, \mathrm{Air}}=\frac{n\left({ }^{18} \mathrm{O}\right)_{\mathrm{B}} / n\left({ }^{16} \mathrm{O}\right)_{\mathrm{B}}-n\left({ }^{18} \mathrm{O}\right)_{\mathrm{Air}} / n\left({ }^{16} \mathrm{O}\right)_{\mathrm{Air}}}{n\left({ }^{18} \mathrm{O}\right)_{\mathrm{Air}} / n\left({ }^{16} \mathrm{O}\right)_{\mathrm{Air}}}=\frac{r\left({ }^{18} \mathrm{O}\right)_{\mathrm{B}}-r\left({ }^{18} \mathrm{O}\right)_{\mathrm{Air}}}{r\left({ }^{18} \mathrm{O}\right)_{\text {Air }}}
$$

Thus, atmospheric $\mathrm{O}_{2}$ will join reference Vienna Standard Mean Ocean Water (VSMOW) and Vienna Pee Dee Belemnite (VPDB) as an international measurement standard for reporting $\delta\left({ }^{17} \mathrm{O}\right)$ and $\delta\left({ }^{18} \mathrm{O}\right)$ values. To be consistent with other expressions of $\delta\left({ }^{18} \mathrm{O}\right)$ with respect to VSMOW or VPDB [36], $\delta\left({ }^{18} \mathrm{O}\right)$ values expressed relative to atmospheric $\mathrm{O}_{2}$ should be given on a scale normalized such that the $\delta\left({ }^{18} \mathrm{O}\right)$ of the international measurement Standard Light Antarctic Precipitation (SLAP) is $-55.5 \%$ relative to VSMOW. The $\delta\left({ }^{18} \mathrm{O}\right)$ of atmospheric $\mathrm{O}_{2}$ (with respect to the VSMOW-SLAP scale) has been reported as $+23.88 \pm 0.03 \%$ [35] and $+23.8 \pm 0.3 \%$ [37]. For $\delta\left({ }^{17} \mathrm{O}\right)$ values, the $\delta\left({ }^{17} \mathrm{O}\right)$ value of SLAP relative to VSMOW should be provided in reports of authors so that scale normalization can be performed by readers if needed.

\section{RECOMMENDATION FOR A NEW BEST MEASUREMENT OF $n\left({ }^{40} \mathrm{Ar}\right) / n\left({ }^{36} \mathrm{Ar}\right)$}

The Commission-recommended value for the isotope-amount ratio of Ar isotopes $n\left({ }^{40} \mathrm{Ar}\right) / n\left({ }^{36} \mathrm{Ar}\right)$ in air has been changed to 298.56(31) from 296.03(53) on the basis of new partially calibrated measurements [38]. The corresponding atomic weight from atmospheric $\mathrm{Ar}$ is indistinguishable from the previous standard atomic weight for Ar of 39.948(1) for which the uncertainty accommodates for the variability. 
The new isotope-amount ratio from Lee et al. [38] is $1 \%$ higher than the value accepted previously. This is significant in the age dating of geological systems using the Ar-K-Ca decay scheme. An accurate determination of an age depends on a reliable $n\left({ }^{40} \mathrm{Ar}\right) / n\left({ }^{36} \mathrm{Ar}\right)$ isotope-amount ratio of the initial argon present before the mineral became isolated and the geological clock began "ticking". Often, $\mathrm{Ar}-\mathrm{K}-\mathrm{Ca}$ dating calculations employ the $n\left({ }^{40} \mathrm{Ar}\right) / n\left({ }^{36} \mathrm{Ar}\right)$ isotope-amount ratio from Nier of $295.5(5)$ [39]. An inaccurate value for the isotope composition of the initial Ar present in the mineral would result in a systematic offset to argon ages.

\section{RECOMMENDATIONS FOR THE INCLUSION OF SYSTEMATIC ERRORS IN THE PUBLICATION OF MASS SPECTROMETRIC ISOTOPE-RATIO MEASUREMENTS}

The recommendations below are presented so that individuals engaged in mass spectrometric isotoperatio measurements can ensure that their publications include all of the information needed for a systematic evaluation of isotope-ratio-measurement uncertainty by CIAAW. The primary task of CIAAW is the biennial evaluation of published isotope-ratio measurements to identify elements whose standard atomic weight and uncertainty can be revised. The exclusive basis of these CIAAW decisions is the information that is provided in the original peer-reviewed publication describing the measurement. CIAAW and SIAM use only peer-reviewed published information. Mass spectrometric measurements are a complex task and are subject both to systematic and statistical errors in the measurement itself and in the various steps involved in the preparation of the measurement and the evaluation of measurement data. To fulfill its task, CIAAW needs to assess the quality of a published measurement, specifically by (a) evaluating possible sources of errors in the measurements and their magnitude and (b) evaluating if sources of error have been properly considered by the authors and propagated to the primary measurement result, i.e., the published isotope ratios. From the reported isotope ratios, isotopic abundances can be calculated and multiplied with the nuclide masses to obtain the atomic weight of the element in the analyzed sample. Based on this assessment, SIAM/CIAAW makes a judgment in how far reported uncertainties in the measurement need to be expanded in order to ensure that the "true" value of the atomic weight of the element in the analyzed sample(s) lies within the margins set by the final uncertainty statement published together with the standard atomic weight. For elements showing substantial natural variations in isotopic abundances, this uncertainty may have to be expanded beyond purely measurement-related uncertainties in the underlying isotope-amount ratios or isotopic abundances.

From this, it becomes apparent that the successful work of CIAAW depends largely on the availability of all information that is necessary for a comprehensive evaluation of a reported isotope-amountratio measurement in the literature. Sources of errors in isotope-amount-ratio measurements can be random or systematic. Authors normally account for random errors, but some systematic errors may be unaccounted for when looking at the data from a CIAAW perspective. This might be because sources of error were unimportant for the topic of the publication or sources of error were overlooked. For the Commission, a proper uncertainty evaluation considering both random and potential systematic errors is important for transparency and consistency of its published data. Potential systematic errors in the measurement will be accounted for by expanding the uncertainty in the measurement symmetrically. From the corrected isotope-amount ratios, both isotope abundances and atomic weights can be calculated.

An evaluation of isotope abundance data by the Commission generally proceeds as follows:

1. Potential best measurement publications are selected by SIAM.

2. SIAM makes a review and extracts measurement results following these guidelines. The published isotope-amount ratios and their associated uncertainties are transferred to an element evaluation form. To allow combination with other data, the reported uncertainties must be converted to standard uncertainties. To convert to standard uncertainties, SIAM follows the recommendations in the JCGM Guide to the Expression of Uncertainty in Measurement (GUM) [41]. The re- 
ported isotope-amount ratios are evaluated and, if needed, uncertainties are expanded to cover possible systematic errors in the measurements. Reported values are corrected only if there is sufficient information in the publication for reliable estimation of systematic offsets. Details on the mathematical approach for expanding uncertainties of published isotope ratios are given below.

3. After calculation using GUM Workbench software, isotope-amount ratios, molar fractions, and the atomic weight with their (expanded) standard uncertainties are exported back to the element database.

For evaluating uncertainties of published isotope-amount ratios, the following sources of systematic errors, at a minimum, should be considered where $x$ represents possible systematic offsets and $k$ represents the corresponding correction factor:

1. System linearity

2. Baseline correction

3. Isobaric interferences

4. Instrumental fractionation

5. Sample preparation

$\begin{array}{ll}x_{l} & k_{l} \\ x_{b} & k_{b} \\ x_{i} & k_{i} \\ x_{f} & k_{f} \\ x_{p} & k_{p}\end{array}$

For the correction of systematic offsets, a correction factor is introduced that carries an uncertainty $k=x \pm u(x)$ where $k$ is the correction factor for a potential systematic error and $u(x)$ is the uncertainty of the correction factor. Both $k$ and $u(x)$ may be different for individual isotope ratios of an element. Unless it is possible to calculate or estimate a reliable systematic offset from published isotope ratios, $x$ will be set to 1 . When considering the above sources of uncertainty, the corrected isotope-number ratio $R$ can be expressed as

$$
R=r \cdot k_{l} \cdot k_{b} \cdot k_{i} \cdot k_{f} \cdot k_{p}
$$

where $r$ is the published isotope-amount ratio.

Applying the laws of error propagation, the relative uncertainty of $R,(u(R) / R)$, is given by

$$
\frac{u(R)}{R}=\sqrt{\left(\frac{u(r)}{r}\right)^{2}+\left(\frac{u\left(k_{l}\right)}{k_{l}}\right)^{2}+\left(\frac{u\left(k_{b}\right)}{k_{b}}\right)^{2}+\left(\frac{u\left(k_{i}\right)}{k_{i}}\right)^{2}+\left(\frac{u\left(k_{f}\right)}{k_{f}}\right)^{2}+\left(\frac{u\left(k_{p}\right)}{k_{p}}\right)^{2}}
$$

Because no reported isotope-ratio value will be revised unless the magnitude of a systematic offset in the data can be assessed from published information, $k$ always equals 1 .

The Commission considers the following systematic offsets when reviewing publications. As far as possible, the measurement modes or measurement conditions are listed in descending order in relation to the possible magnitude of the offset.

1. Offsets due to system nonlinearity $\left(k_{l}\right)$

- fully calibrated measurement (including a linearity check)

- Faraday cup(s) for ion detection

- $\quad$ single collector

- multicollector (use of a virtual amplifier system; correction for differences in cup efficiency; use of gain factors for each individual cup)

- $\quad$ secondary electron multiplier(s) for ion detection (single ion counting, continuous mode)

2. Offsets due to baseline effects $\left(k_{b}\right)$

- $\quad$ detector system (Faraday cup(s) or secondary electron multipliers)

- measurement mode (on peak/between peaks, closed valve/open valve, between scans/blocks/measurements) 
3. Offsets due to isobaric interferences $\left(k_{i}\right)$

- $\quad$ correction of known/identified isobaric interferences (TIMS, ICP-MS)

- using a measured isotope-amount ratio to consider natural or instrumental fractionation effects

- $\quad$ use of a tabulated isotope-amount ratio

- ICP-MS measurements

- demonstrated absence of isobaric interferences (three-isotope plots)

- $\quad$ assessment for presence of uncorrected isobaric interferences

- $\quad$ instrument type (high resolution, collision cell, low resolution, etc.)

- $\quad$ separation of element from sample matrix

- $\quad$ direct measurement of samples in complex matrix

4. Offsets due to instrumental fractionation effects $\left(k_{f}\right)$

- fully calibrated measurement

- $\quad$ total evaporation method (TIMS)

- $\quad$ no normalization applied

- $\quad$ internal normalization (TIMS, ICP-MS)

- $\quad$ using some (one or more) self-determined, calibrated isotope-ratio measurements

- $\quad$ using a published isotope ratio from the literature

- $\quad$ external normalization (ICP-MS)

- $\quad$ by standard/sample bracketing (using a certified isotopic reference material; using an uncertified reference material; using matrix-matched samples/standards)

- $\quad$ by normalization to a second, spiked element (using a certified isotopic reference material; using an uncertified consensus reference material; validity of correction technique established; as part of the publication/survey; by adopting findings from other authors)

5. Offsets due to fractionation effects during sample preparation $\left(k_{p}\right)$

- $\quad$ separation of an element from the sample matrix

- monitored by parallel processing of a standard (using a certified isotopic reference material; using a reference material having a consensus value)

- $\quad$ separation procedure and separation yield were checked beforehand

- $\quad$ analysis of shelf reagent/standard without matrix separation

\section{MEMBERSHIP OF SPONSORING BODIES}

Membership of the Inorganic Chemistry Division Committee for the period 2008-2009 was as follows:

President: K. Tatsumi (Japan); Past President: A. R. West (UK); Secretary: L. V. Interrante (USA); Vice President: R. D. Loss (Australia); Titular Members: T. B. Coplen (USA); T. Ding (China/Beijing); J. Garcia-Martinez (Spain); M. Leskelä (Finland); L. A. Oro (Spain); J. Reedijk (Netherlands); M. P. Suh (Korea); Associate Members: A. V. Chadwick (UK); M. Drábik (Slovakia); N. E. Holden (USA); S. Mathur (Germany); K. Sakai (Japan); J. Takats (Canada); National Representatives: T. V. Basova (Russia); A. Bologna Alles (Uruguay); R. Gonfiantini (Italy); P. Karen (Norway); L.-K. Liu (China/Taipei); L. R. Ohrström (Sweden).

Membership of the Commission on Isotopic Abundances and Atomic Weights for the period 2008-2009 was as follows:

Chair: T. P. Ding (China); Secretary: M. E. Wieser (Canada); Titular Members: M. Berglund (Belgium); T. Walczyk (Switzerland); S. Yoneda (Japan); Associate Members: R. Gonfiantini (Italy); M. Gröning (Austria); H. Hidaka (Japan); X.-K. Zhu (China); National Representatives: J. K. Böhlke (USA); P. De Bièvre (Belgium); J. de Laeter (Australia). 
Membership of the Task Group on Evaluated Published Isotope Ratio Data (2005-2007) was as follows:

Chair: M. Berglund (Belgium); Members: T. B. Coplen (USA); P. De Bièvre (Belgium); J. R. de Laeter (Australia); T. Ding (China/Beijing); R. Gonfiantini (Italy); M. Gröning (Austria); N. E. Holden (USA); R. D. Loss (Australia); K. J. R. Rosman (Australia, deceased); E. Roth (France, deceased); T. R. Walczyk (Switzerland); M. Wieser (Canada); M.-t. Zhao (China/Beijing); X. Zhu (China/Beijing).

The Commission on Isotopic Abundances and Atomic Weights notes the death of two former members of the Commission. Harry J. Svec, 24 June 1918 to 28 November 2006, developed the isotopic reference material used for lithium measurements called L-Svec. Harry was an Associate Member of the Commission from 1967 to 1971. Aaldert H. Wapstra, 24 April 1923 to 2 December 2006, published, on behalf of IUPAP, the Atomic Mass Table that is used to calculate the atomic weight values of the chemical elements for the last half-century. Aaldert was a Titular Member, as well as an Associate Member, of the Commission from 1963 to 1979.

\section{REFERENCES}

1. M. E. Wieser. Pure Appl. Chem. 78, 2051 (2006).

2. M. E. Wieser. J. Phys. Chem. Ref. Data 36, 485 (2007).

3. J. K. Böhlke, J. R. DeLaeter, P. De Bièvre, H. Hidaka, H. S. Peiser, K. J. R. Rosman, P. D. P Taylor. J. Phys. Chem. Ref. Data 34, 57 (2005).

4. H. S. Peiser, N. E. Holden, P. De Bièvre, I. L. Barnes, R. Hagemann, J. R. De Laeter, T. J. Murphy, E. Roth, M. Shima, H. G. Thode. Pure Appl. Chem. 56, 695 (1984).

5. J. R. De Laeter, J. K. Böhlke, P. De Bièvre, H. Hidaka, H. S. Peiser, K. J. R. Rosman, P. D. P. Taylor. Pure Appl. Chem. 75, 683 (2003).

6. G. Audi, A. H. Wapstra, C. Thibault. Nucl. Phys. A 729, 337 (2003).

7. T. B. Coplen, H. S. Peiser. Pure Appl. Chem. 70, 237 (1998).

8. (a) F. W. Clarke. J. Am. Chem. Soc. 16, 179 (1894); (b) F. W. Clarke. J. Am. Chem. Soc. 17, 201 (1895); (c) F. W. Clarke. J. Am. Chem. Soc. 18, 197 (1896); (d) F. W. Clarke. J. Am. Chem. Soc. 19, 359 (1897); (e) F. W. Clarke. J. Am. Chem. Soc. 20, 163 (1898); (f) F. W. Clarke. J. Am. Chem. Soc. 21, 200 (1899); (g) F. W. Clarke. J. Am. Chem. Soc. 22, 70 (1900).

9. IUPAC. Nomenclature of Inorganic Chemistry, IUPAC Recommendations 2005 (the "Red Book"), prepared for publication by N. Connelly, T. Damhus, R. M. Harshorn, RSC Publishing, Cambridge, UK (2005).

10. J. R. DeLaeter, N. Bukilic. Phys. Rev. C 73, 045806-1 (2006).

11. J. R. DeLaeter, N. Bukilic. Int. J. Mass Spectrom. 252, 222 (2006).

12. M. T. McCulloch, J. R. DeLaeter, J. J. R. Rosman. Earth Planet. Sci. Lett. 28, 308 (1976).

13. M. E. Wieser, J. R. De Laeter. Phys. Rev. C 75, 55802-1 (2007).

14. R. D. Loss. Pure Appl. Chem. 75, 1107 (2003).

15. O. Hönigschmid, G. Wittmann. Z. Anorg. Allg. Chem. 229, 65 (1936).

16. M. Tanimizu, T. J. Hirata. Anal. Atom. Spectrosc. 21, 1423 (2006).

17. R. J. Hayden, D. C. Hess, M. G. Inghram. Phys. Rev. 75, 322 (1950).

18. W. T. Leland. Phys. Rev. 77, 634 (1950).

19. A. E. Cameron, E. Wichers. J. Am. Chem. Soc. 84, 4175 (1962).

20. E. Ponzevera, C. Quetel, M. Bergland, P. Taylor, P. Evans, R. D. Loss, G. Fortunato. J. Am. Soc. Mass Spectrom. 17, 1412 (2006).

21. T.-L. Chang, M.-T. Zhao, W.-J. Li, J. Wang, Q.-Y. Qian. Int. J. Mass Spectrom. 208, 113 (2001).

22. M. Tanimizu, Y. Asada, T. Hirata. Anal. Chem. 74, 5814 (2002).

23. G. Marinenko, R. T. Foley. J. Res. Natl. Bur. Stand. A 75, 561 (1970).

24. K. J. R. Rosman. Geochim. Cosmochim. Acta 36, 801 (1972). 
25. W. T. Leyland, A. O. Nier. Phys. Rev. 73, 1206 (1948).

26. D. C. Hess, M. G. Inghram Jr., R. J. Hayden. Phys. Rev. 74, 1531 (1948).

27. K. T. Bainbridge, A. O. Nier. Preliminary Report, National Research Council, Washington, p. 9 (1950)

28. R. F. Hibbs. Report AECU-556 (1949).

29. J. Okamoto, M. Kakuta, N. Morito, Y. Nakajima, H. Tsuyama, H. Onuki. Jpn. Analyst 8, 445 (1959).

30. F. Konishi, K. Kusao, N. Nakamura. Shitsuryo Bunseki 14, 275 (1966).

31. N. E. Holden. Pure Appl. Chem. 61, 1483 (1989).

32. N. E. Holden. Pure Appl. Chem. 62, 941 (1990).

33. N. E. Holden. "Table of the isotopes", in CRC Handbook of Chemistry and Physics, $79^{\text {th }}$ ed., sect. 11, pp. 41-140, CRC Press, Boca Raton (1998) and updates.

34. N. E. Holden, D. C. Hoffman. Pure Appl. Chem. 72, 1525 (2000).

35. E. Barkan, B. Luz. Rapid Commun. Mass Spectrom. 19, 3737 (2005).

36. T. B. Coplen. Pure Appl. Chem. 66, 273 (1994).

37. T. B. Coplen, J. A. Hopple, J. K. Böhlke, H. S. Peiser, S. E. Rieder, H. R. Krouse, K. J. R. Rosman, T. Ding, R. D. Vocke, Jr., K. M. Révész, A. Lamberty, P. Taylor, P. De Bièvre. Compilation of minimum and maximum isotope ratios of selected elements in naturally occurring terrestrial materials and reagents: U.S. Geological Survey Water-Resources Investigations Report 01-4222 (2001).

38. J.-Y. Lee, K. Marti, J. P. Severinghaus, K. Kenji, H.-S. Yoo, J. B. Lee, J. S. Kim. Geochim. Cosmochim. Acta 70, 4507 (2006).

39. A. O. Nier. Phys. Rev. 77, 789 (1950).

40. S. S. Assonov, C. A. M. Brenninkmeijer. Rapid Commun. Mass Spectrom. 17, 1007 (2003).

41. JCGM 100:2008 (GUM 1995 with minor corrections), Evaluation of measurement data - Guide to the expression of uncertainty in measurement. Joint Committee on Guides to Measurement (2008).

Republication or reproduction of this report or its storage and/or dissemination by electronic means is permitted without the need for formal IUPAC permission on condition that an acknowledgment, with full reference to the source, along with use of the copyright symbol $\odot$, the name IUPAC, and the year of publication, are prominently visible. Publication of a translation into another language is subject to the additional condition of prior approval from the relevant IUPAC National Adhering Organization. 